\title{
THE XMM-NEWTON WIDE-FIELD SURVEY IN THE COSMOS FIELD (XMM-COSMOS): DEMOGRAPHY AND MULTIWAVELENGTH PROPERTIES OF OBSCURED AND UNOBSCURED LUMINOUS ACTIVE GALACTIC NUCLEI*
}

M. Brusa ${ }^{1}$, F. Civano ${ }^{2}$, A. Comastri ${ }^{3}$, T. Miyaji ${ }^{4,5}$, M. Salvato ${ }^{6,7,8}$, G. Zamorani $^{3}$, N. CAPPelluti $^{1,9}$, F. Fiore $^{10}$, G. Hasinger $^{6}$, V. Mainieri ${ }^{11}$, A. Merloni ${ }^{1,7}$, A. Bongiorno ${ }^{1}$, P. CapaK ${ }^{12}$, M. Elvis ${ }^{2}$, R. Gilli ${ }^{3}$, H. HaO $^{2}$, K. JahnKe $^{13}$, A. M. KoeKemoer ${ }^{14}$, O. Ilbert ${ }^{15}$, E. Le Floc'H ${ }^{16}$, E. Lusso $^{3,17}$, M. Mignoli ${ }^{3}$, E. SchinnereR ${ }^{13}$, J. D. Silverman $^{18}$, E. Treister ${ }^{19}$, J. D. Trump ${ }^{20}$, C. Vignali ${ }^{3,17}$, M. Zamojski ${ }^{12}$, T. Aldcroft ${ }^{2}$, H. Aussel ${ }^{21}$, S. Bardelli $^{3}$, M. Bolzonella $^{3}$,

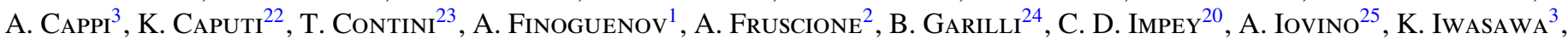
P. KampCZYK ${ }^{26}$, J. KartaltePe ${ }^{19,25}$, J. P. KNeIB ${ }^{27}$, C. KNOBEL ${ }^{26}$, K. KovaC ${ }^{26}$, F. Lamareille ${ }^{23}$, J.-F. Leborgne $^{23}$, V. Le Brun ${ }^{27}$, O. Le Fevre ${ }^{27}$, S. J. Lilly ${ }^{26}$, C. Maier ${ }^{26}$, H. J. McCracken ${ }^{28}$, R. Pello ${ }^{23}$, Y.-J. Peng ${ }^{26}$, E. Perez-Montero ${ }^{26}$,

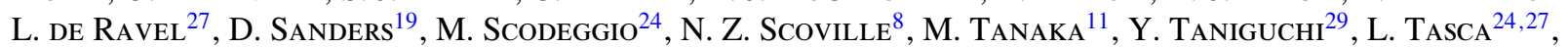
S. DE LA TORRE ${ }^{27}$, L. TRESSE $^{27}$, D. VERGANi ${ }^{3}$, AND E. ZuCCA ${ }^{3}$

${ }^{1}$ Max-Planck-Institut für extraterrestrische Physik, Giessenbachstrasse 1, D-85748, Garching bei München, Germany

${ }^{2}$ Harvard-Smithsonian Center for Astrophysics, 60 Garden Street, Cambridge, MA 02138, USA

${ }^{3}$ INAF-Osservatorio Astronomico di Bologna, via Ranzani 1, I-40127, Bologna, Italy

${ }^{4}$ Instituto de Astronomía, Universidad Nacional Autónoma de México, Ensenada, Mexico

${ }^{5}$ Center for Astrophysics and Space Sciences, University of California at San Diego, Code 0424, 9500 Gilman Drive, La Jolla, CA 92093, USA

${ }^{6}$ IPP-Max-Planck-Institute for Plasma Physics, Boltzmannstrasse 2, D-85748, Garching, Germany

${ }^{7}$ Excellence Cluster Universe, Boltzmannstrasse 2, D-85748, Garching bei Muenchen, Germany

${ }^{8}$ California Institute of Technology, 1201 East California blvd, Pasadena, CA 91125, USA

${ }^{9}$ University of Maryland, Baltimore County, 1000 Hilltop Circle, Baltimore, MD 21250, USA

${ }^{10}$ INAF-Osservatorio Astronomico di Roma, via Frascati 33, Monteporzio-Catone (Roma), I-00040, Italy

${ }^{11}$ European Southern Observatory, Karl-Schwarzschild-str. 2, 85748, Garching bei München, Germany

${ }^{12}$ Spitzer Science Center, California Institute of Technology, MC 220-6, 1200 East California Boulevard, Pasadena, CA 91125, USA

${ }^{13}$ Max-Planck-Institut für Astronomie, Königstuhl 17, D-69117, Heidelberg, Germany

${ }^{14}$ Space Telescope Science Institute, 3700 San Martin Drive, Baltimore, MD 21218, USA

${ }^{15}$ Laboratoire d'Astrophysique de Marseille, BP 8, Traverse du Siphon, 13376 Marseille Cedex 12, France

${ }^{16}$ Laboratoire AIM, CEA/DSM-CNRS-Université Paris Diderot, DAPNIA/Service d'Astrophysique, Bât. 709, CEA-Saclay, F-91191 Gif-sur-Yvette Cédex, France

${ }^{17}$ Dipartimento di Astronomia, Università di Bologna, via Ranzani 1, 40127, Bologna, Italy

${ }^{18}$ Institute for the Physics and Mathematics of the Universe (IPMU), University of Tokyo, Kashiwanoha 5-1-5, Kashiwa, Chiba 277-8568, Japan

${ }^{19}$ Institute for Astronomy, University of Hawaii, 2680 Woodlawn Drive, Honolulu, HI 96822, USA

${ }^{20}$ Steward Observatory, University of Arizona, 933 North Cherry Avenue, Tucson, AZ 85 721, USA

${ }^{21}$ Institut d'Astrophysique de Paris, UMR7095 CNRS, Université Pierre \& Marie Curie, 98 bis boulevard Arago, 75014 Paris, France

${ }^{22}$ SUPA Institute for Astronomy, The University of Edinburgh, Royal Observatory, Blackford Hill, Edinburgh EH9 3HJ, UK

${ }^{23}$ Observatoire Midi-Pyrénées, Laboratoire d'Astrophysique (UMR 5572), 14 Avenue E. Belin, F-31400 Toulouse, France

${ }^{24}$ INAF IASF-Milano, via Bassini 15, 20133, Milano, Italy

${ }^{25}$ National Optical Astronomy Observatory, 950 North Cherry Avenue, Tucson, AZ 85 721, USA

${ }^{26}$ Department of Physics, ETH Zurich, CH-8093 Zurich, Switzerland

${ }^{27}$ Laboratoire d'Astrophysique de Marseille, Traverse du Siphon, F-13376 Marseille, France

${ }^{28}$ Observatoire de Paris, LERMA, 61 Avenue de l'Observatoire, 75014 Paris, France

${ }^{29}$ Physics Department, Graduate School of Science and Engineering, Ehime University, 2-5 Bunkyo-cho, Matsuyama, Ehime 790-8577, Japan Received 2009 December 23; accepted 2010 April 15; published 2010 May 18

\section{ABSTRACT}

We report the final optical identifications of the medium-depth ( $\sim 60 \mathrm{ks})$, contiguous $\left(2 \mathrm{deg}^{2}\right)$ XMM-Newton survey of the COSMOS field. XMM-Newton has detected $\sim 1800$ X-ray sources down to limiting fluxes of $\sim 5 \times 10^{-16}, \sim 3$ $\times 10^{-15}$, and $\sim 7 \times 10^{-15} \mathrm{erg} \mathrm{cm}^{-2} \mathrm{~s}^{-1}$ in the $0.5-2 \mathrm{keV}, 2-10 \mathrm{keV}$, and 5-10 keV bands, respectively $\left(\sim 1 \times 10^{-15}\right.$, $\sim 6 \times 10^{-15}$, and $\sim 1 \times 10^{-14} \mathrm{erg} \mathrm{cm}^{-2} \mathrm{~s}^{-1}$, in the three bands, respectively, over $50 \%$ of the area). The work is complemented by an extensive collection of multiwavelength data from $24 \mu \mathrm{m}$ to UV, available from the COSMOS survey, for each of the X-ray sources, including spectroscopic redshifts for $\gtrsim 50 \%$ of the sample, and high-quality photometric redshifts for the rest. The $X M M$ and multiwavelength flux limits are well matched: $1760(98 \%)$ of the X-ray sources have optical counterparts, $1711(\sim 95 \%)$ have IRAC counterparts, and $1394(\sim 78 \%)$ have MIPS $24 \mu$ m detections. Thanks to the redshift completeness (almost 100\%) we were able to constrain the high-luminosity tail of the X-ray luminosity function confirming that the peak of the number density of $\log L_{X}>44.5$ active galactic nuclei (AGNs) is at $z \sim 2$. Spectroscopically identified obscured and unobscured AGNs, as well as normal and star-forming galaxies, present well-defined optical and infrared properties. We devised a robust method to identify a sample of $\sim 150$ high-redshift $(z>1)$, obscured AGN candidates for which optical spectroscopy is not available. We were able to determine that the fraction of the obscured AGN population at the highest $\left(L_{X}>10^{44} \mathrm{erg} \mathrm{s}^{-1}\right)$ $\mathrm{X}$-ray luminosity is $\sim 15 \%-30 \%$ when selection effects are taken into account, providing an important observational constraint for X-ray background synthesis. We studied in detail the optical spectrum and the overall spectral energy 
distribution of a prototypical Type 2 QSO, caught in a stage transitioning from being starburst dominated to AGN dominated, which was possible to isolate only thanks to the combination of X-ray and infrared observations.

Key words: galaxies: active - surveys - X-rays: diffuse background - X-rays: galaxies - X-rays: general

Online-only material: color figures, machine-readable table

\section{INTRODUCTION}

First introduced to explain the properties we observe today in normal galaxies such as their color bimodality (Blanton et al. 2003; Bell et al. 2004; Faber et al. 2007) and the so-called local scaling relations (Ferrarese \& Merrit 2000; Gebhardt et al. 2000; Gültekin et al. 2009), the existence of a "feedback" between the accreting super massive black hole (SMBH) and the host galaxy in which it resides ultimately challenged our understanding of active galactic nuclei (AGNs). Indeed, the widely accepted scenario of galaxy-AGN coevolution (e.g., Silk \& Rees 1998; Fabian \& Iwasawa 1999; Granato et al. 2004; Di Matteo et al. 2005; Menci et al. 2008) points toward a physical coupling responsible for the self-regulated SMBH and galaxy growth. In particular, according to the theoretical scenario emerging from extensive semianalytical models and hydrodynamics simulations, a key event in the SMBH versus host-galaxy coevolution is represented by the highly obscured AGN phase, when large quantities of gas driven to the center in the merger of two disk galaxies were available to efficiently feed and obscure the growing black hole and build galaxy stellar mass through significant episodes of star formation. This phase ends when strong winds and shocks from the central AGN heat the interstellar medium, blowing away the dust and gas, thus cleaning the line of sight and inhibiting further star formation (see, e.g., Granato et al. 2004; Di Matteo et al. 2005; Croton et al. 2006; Sijacki et al. 2007; Menci et al. 2008; Hopkins et al. 2008).

In this general framework for merger-induced AGN feedback, capable of passing numerous observational tests (e.g., Sanders et al. 1988; Hopkins et al. 2006; Veilleux et al. 2009), the differences between "obscured" and "unobscured" AGNs are no longer uniquely described under a geometrical unification model (in which they are simply related to orientation effects; Antonucci \& Miller 1985; Antonucci 1993; Urry \& Padovani 1995), but can be interpreted as due to the fact that the same objects are observed in different evolutionary phases. This hypothesis is consistent with the finding, mainly from $\mathrm{X}$-ray surveys, that absorption is much more common at low luminosities (see, e.g., Ueda et al. 2003; La Franca et al. 2005; Maiolino et al. 2007; Hasinger 2008) and, possibly, at high redshift (La Franca et al. 2005; Ballantyne et al. 2006; Treister \& Urry 2006; Hasinger 2008). The luminosity and redshift dependence of the obscuring fraction may be naturally linked to the AGN radiative power (related to the intrinsic X-ray

\footnotetext{
* Based on data collected at: the NASA/ESA Hubble Space Telescope, obtained at the Space Telescope Science Institute, which is operated by AURA Inc, under NASA contract NAS 5-26555; the Subaru Telescope, which is operated by the National Astronomical Observatory of Japan; the European Southern Observatory, Chile, under Large Program 175.A-0839; Kitt Peak National Observatory, Cerro Tololo Inter-American Observatory, and the National Optical Astronomy Observatory, which are operated by the Association of Universities for Research in Astronomy, Inc. (AURA) under cooperative agreement with the National Science Foundation; and the Canada-France-Hawaii Telescope operated by the National Research Council of Canada, the Centre National de la Recherche Scientifique de France and the University of Hawaii.
}

luminosity; see, e.g., Lawrence \& Elvis 1982) which is able to ionize and expel gas (more common at high- $z$ ) and dust from the nuclear regions, nicely fitting the current framework of AGN formation and evolution sketched above (see, for example, Hopkins et al. 2006). The complete picture is likely to be more complex, depending on many other parameters such as the $\mathrm{BH}$ mass, the Eddington ratio, the star formation activity in the host galaxy and, in particular, the timescales associated with the AGN duty cycle activity.

A correct and complete identification of unobscured, obscured, and highly obscured AGNs at all redshifts (and especially in the $z=1-3$ interval, where most of the feedback is expected to happen) is therefore crucial for a comprehensive understanding of the still little explored phase of the common growth of SMBHs and their host galaxies. While it is relatively straightforward to select unobscured AGNs from optical multicolor surveys (e.g., Richards et al. 2002) and/or from spectroscopic samples (e.g., the VVDS survey; Gavignaud et al. 2006; Bongiorno et al. 2007), the most efficient, reddening independent method to select obscured, Type 2 AGNs is the presence of luminous X-ray emission $\left(L_{[2-10 \mathrm{keV}]}>10^{42} \mathrm{erg} \mathrm{s}^{-1}\right)$ and hard $\mathrm{X}$-ray colors (see Brandt \& Hasinger 2005 for a review). Indeed, hard $(2-10 \mathrm{keV}) \mathrm{X}$-ray nuclear emission is an almost unambiguous mark of the presence of an AGN, given the extremely small contamination from star formation induced emission at these luminosities and frequencies. Combined with the fact that hard $\mathrm{X}$-rays are not seriously affected by obscuration due to neutral gas along the line of sight (up to $N_{\mathrm{H}} \sim 10^{23} \mathrm{~cm}^{-2}$ ), it is clear that hard X-ray selection is the most effective method to uncover unobscured to moderate obscured AGNs and study in detail their demographics (see Comastri \& Brusa 2008; Brandt $\&$ Alexander 2010 for a review).

Since the launch in 1999 of both the XMM-Newton and Chandra satellites, a large ( $>30)$ number of surveys covering a wide fraction of the area versus depth plane (see Figure 1 in Brandt \& Hasinger 2005; see also Hickox 2009) have been performed, and our understanding of AGN properties and evolution has received a major boost. Thanks to vigorous programs of multiwavelength follow-up campaigns, sensitive $\mathrm{X}$-ray observations turned out to be highly efficient in unveiling weak and/or "elusive" accreting black holes, in a variety of otherwise non-active galaxies (i.e., not recognized as AGNs from the optical spectra or continuum emission), such as (among others) X-ray bright optically normal galaxies (XBONGs; e.g., Comastri et al. 2002; Severgnini et al. 2003; Civano et al. 2007), extremely red objects (e.g., Alexander et al. 2002; Brusa et al. 2005; Severgnini et al. 2005), submillimeter galaxies (e.g., Alexander et al. 2005; Laird et al. 2010), high-z starforming systems (e.g., Daddi et al. 2007; Fiore et al. 2008, 2009; Treister et al. 2009b), Lyman break galaxies (LBGs; e.g., Brandt et al. 2001; Nandra et al. 2002; Aird et al. 2008). In many of these cases, the AGN responsible for the X-ray emission is overwhelmed at longer wavelength by the host-galaxy light and/ or the obscuration might be connected to processes within the host galaxy itself, such as the presence of dust lanes or starburst 
disks (see, e.g., Martinez-Sansigre et al. 2005; Ballantyne et al. 2006; Ballantyne 2008; Hopkins et al. 2009). This suggests that the accretion activity (especially in high-redshift sources) can be unambiguously revealed thanks to the presence of a strong X-ray emission (see, e.g., discussion in Brusa et al. $2009 \mathrm{~b}$ ) and, therefore, the combination of both X-ray and optical classifications, coupled with the multiwavelength analysis, can be crucial to fully assess the nature of the candidate AGN.

The high level of completeness in redshift determination for a large number of X-ray selected AGNs (up to a few thousands) has made possible a robust determination of the luminosity function and evolution of unobscured and mildly obscured AGNs which turned out to be luminosity dependent: the space density of bright QSOs $\left(L_{X}>10^{44} \mathrm{erg} \mathrm{s}^{-1}\right)$ peaks at $z \sim 2-3$, to be compared with the $z \sim 0.7-1$ peak of lower-luminosity Seyfert galaxies (Ueda et al. 2003; Hasinger et al. 2005; La Franca et al. 2005; Silverman et al. 2008; Ebrero et al. 2009; Yencho et al. 2009). Based on the Ueda et al. (2003) work, Marconi et al. (2004) and Merloni (2004) were the first to propose that SMBH undergo a "antihierarchical" evolution, in the form of a differential growth (earlier and faster for more massive black holes). This antihierarchical behavior observed in AGN evolution (similar to that observed in normal galaxies; e.g., Cowie et al. 1996) provided an important and independent confirmation that the formation and evolution of SMBH and their host galaxies are likely different aspects of the same astrophysical process. It should be noted that the luminosity-dependent density evolution (LDDE) parameterization, corresponding to a strong downsizing, has been recently questioned by Aird et al. (2010). Their preferred model for the evolution of the X-ray luminosity function (XLF) is a luminosity and density evolution model (LADE), where the shift in the redshift peak of AGN space density as function of $\mathrm{X}$-ray luminosity is much weaker than in LDDE models.

A full characterization of the AGN bolometric luminosity function can be obtained only exploring the entire area versus flux plane (e.g., combining samples from deep and large area surveys) and adopting different AGN selection methods (X-ray versus optical versus infrared). Nevertheless, it seems plausible that the blow-out phase associated with the obscured growth represents a relatively short, but very powerful, episode in the QSO lifetime. For this reason, the probability to detect rare and luminous X-ray events is maximized by large area surveys and, in particular, by hard X-ray observations (to cope with obscuration effects) and associated deep multiwavelength coverage.

The XMM-Newton wide-field survey in the COSMOS field (hereinafter XMM-COSMOS; Hasinger et al. 2007) is an important step forward in addressing the topics described above. The $\sim 2 \mathrm{deg}^{2}$ area of the HST/ACS COSMOS Treasury program (Scoville et al. 2007a, 2007b; Koekemoer et al. 2007) has been surveyed with XMM-Newton for a total of $\sim 1.55 \mathrm{Ms}$ during $\mathrm{AO} 3, \mathrm{AO} 4$, and AO6 cycles of XMM observations (Cappelluti et al. 2007, 2009, hereafter C09). XMM-COSMOS provides an unprecedently large sample of point-like X-ray sources ( $\gtrsim 1800$ ), detected over a large, contiguous area, with complete ultraviolet to mid-infrared (including Spitzer data) and radio coverage, and extensive spectroscopic follow-up granted through the zCOSMOS (Lilly et al. 2007, 2009) and Magellan/ IMACS (Trump et al. 2007, 2009) projects. The excellent multiband photometry available in this area allows a robust photometric redshift estimate for the faint sources not reachable by optical spectroscopy, thus allowing a virtually complete sample of X-ray sources. The XMM-COSMOS project is described in Hasinger et al. (2007), while the X-ray point source catalog and counts from the complete XMM-COSMOS survey are presented in a companion paper (C09). The present paper, which extends the work presented by Brusa et al. (2007, hereafter B07) on the optical identifications of the X-ray point sources in the XMM-COSMOS survey, discusses the multiwavelength properties of this large sample of X-ray selected AGNs. Several works have already appeared in the literature and are based on this catalog, or on previous versions of it, e.g., the derivation of AGN photometric redshifts (Salvato et al. 2009), the IMACS AGN spectroscopic campaign (Trump et al. 2009), the clustering properties of spectroscopically confirmed AGNs (Gilli et al. 2009), the space density of high-redshift QSOs (Brusa et al. 2009a), the host-galaxies properties of AGNs in COSMOS (Gabor et al. 2009), the ongoing and coevolving star formation and AGN activity at $z<0.8$ (Silverman et al. 2009a), the environments of AGNs within the galaxies density field (Silverman et al. 2009b), and the relation of the X-ray and optical emission in BL AGNs (Lusso et al. 2010).

The paper is organized as follows: Section 2 presents the multiwavelength data sets drawn from the COSMOS survey and used in the paper; Section 3 describes the method used to identify the X-ray sources and its statistical reliability; the XMM-COSMOS multiwavelength catalog is presented in Section 4, while Section 5 reports the redshift information for the X-ray sources. The number density evolution of luminous XMM-COSMOS sources is presented in Section 6. Section 7 describes the X-ray-to-optical and near-infrared properties of the identified population and of the obscured AGN candidates, while in Section 8 we discuss the obscured AGN fraction as a function of the X-ray luminosity. Section 9 presents the spectrum and spectral energy distribution (SED) of a prototype obscured QSO at $z \sim 1.6$, and in Section 10 we summarize the most important results. Throughout the paper, we adopt the cosmological parameters $H_{0}=70 \mathrm{~km} \mathrm{~s}^{-1} \mathrm{Mpc}^{-1}$, $\Omega_{m}=0.3$, and $\Omega_{\Lambda}=0.7$ (Spergel et al. 2003). In quoting magnitudes, the AB system will be used, unless otherwise stated.

\section{MULTIWAVELENGTH DATA SETS}

\subsection{X-ray}

The catalog used in this work includes 1848 point-like sources above a given threshold with a maximum likelihood detection algorithm in at least one of the soft $(0.5-2 \mathrm{keV})$, hard $(2-10 \mathrm{keV})$, or ultra-hard (5-10 keV) bands down to nominal limiting fluxes of $\sim 5 \times 10^{-16}, \sim 3 \times 10^{-15}$, and $\sim 7 \times 10^{-15} \mathrm{erg} \mathrm{cm}^{-2} \mathrm{~s}^{-1}$, respectively (i.e., the flux of the faintest source detected in the band; see C09). The adopted likelihood threshold corresponds to a probability $\sim 4.5 \times 10^{-5}$ that a catalog source is a spurious background fluctuation (see Cappelluti et al. 2007; C09 for more details). In the present analysis, we used the source list created from 53 out of the 55 XMM-COSMOS fields; for the additional 65, faint sources detected when the pointings obtained in AO6 are included, the identification is not completed yet. For this reason, the number of XMM-COSMOS sources is slightly lower than that discussed in C09. Twenty-six faint sources in our catalog are coincident with diffuse $X M M$ sources in the catalog by Finoguenov et al. (2007). These sources are flagged as "possibly extended" in the point-like catalog and are excluded from the following analysis. The inner part of the COSMOS field has been imaged for a total of 1.8 Ms by Chandra (Elvis et al. 2009), with 36 pointings of $\sim 50 \mathrm{ks}$ each in a $6 \times 6$ array. The mosaic 
covers an area of $\sim 0.92 \mathrm{deg}^{2}$ (about half of the XMM-COSMOS field) down to a limiting flux of $\sim 2 \times 10^{-16} \mathrm{erg} \mathrm{cm}^{-2} \mathrm{~s}^{-1}$ in the soft band and $\sim 7.3 \times 10^{-16} \mathrm{erg} \mathrm{cm}^{-2} \mathrm{~s}^{-1}$ in the hard band, i.e., 3-4 times deeper than XMM-COSMOS. Of the 1822 XMM sources, 945 (51.9\%) have been observed by Chandra with an exposure larger than $30 \mathrm{ks}$, and 875 of them are present in the C-COSMOS point-like source catalog (Elvis et al. 2009; Puccetti et al. 2009). Of the 70 sources not recovered by Chan$d r a$, more than half are in regions with relatively low exposure (between 30 and $50 \mathrm{ks}$ ) and are detected at faint $X M M$ fluxes, mostly in the hard band $\left(\lesssim 6 \times 10^{-15} \mathrm{erg} \mathrm{cm}^{-2} \mathrm{~s}^{-1}\right)$. The remainder are either sources with only hard XMM detections (14) or, after a visual inspection, they can be associated with spurious sources (15), consistent with the expected fraction of spurious sources in the XMM-COSMOS field (see Elvis et al. 2009). Twenty-five of the 875 XMM-COSMOS sources with Chandra detection $(2.8 \%)$ are resolved in two different Chandra sources, lying at distances between 2 and 10 arcsec from each other and therefore being likely blurred in the $X M M$ large point-spread function (PSF; see discussion in $\mathrm{C} 09^{30}$ ). We will further discard these 25 sources in the following analysis. The XMMCOSMOS sample presented in this catalog consists therefore of 1797 X-ray sources, 850 with Chandra detection (47.3\%). In the following, we use the subarcsec accurate Chandra positions to control-check the optical/NIR identifications proposed in Section 3, and to assess the reliability of the proposed identifications.

\subsection{Optical, Near-infrared and Spitzer Photometry}

As we will describe in the next section, the XMM-COSMOS catalog has been cross-correlated with an updated version of the optical multiband catalog of Capak et al. 2007 ("optical catalog" hereafter), the Canada-France-Hawaii Telescope (CFHT)/ $K$-band catalog (McCracken et al. 2010; " $K$-band catalog” hereafter), the IRAC catalog (Sanders et al. 2007; Ilbert et al. 2009; "IRAC catalog" hereafter), and the $24 \mu \mathrm{m}$ MIPS catalog (Le Floc'h et al. 2009; "MIPS catalog" hereafter). The optical cat$\operatorname{alog}^{31}$ contains about 1.5 million objects detected in at least one of the Subaru bands $(b, v, g, r, i, z)$ down to an AB magnitude limit of $\sim 27$. The $K$-band catalog contains about $5 \times 10^{5}$ galaxies detected at a signal-to-noise ratio $(\mathrm{S} / \mathrm{N})>5$ down to $K(\mathrm{AB})=23.5$ (see details in McCracken et al. 2010). The IRAC catalog contains about $4 \times 10^{5}$ objects detected in the $3.6 \mu \mathrm{m}$ (IRAC channel 1) band and it is $90 \%$ complete at $>1 \mu \mathrm{Jy}$ $(\mathrm{AB}=23.9)$. For each source in the IRAC catalog, the photometry from all the other IRAC channels is also reported. The MIPS catalog, obtained in cycles 2 and 3, has very accurate photometry (Sanders et al. 2007). The catalog has been cleaned

\footnotetext{
30 The twenty-five XMM-COSMOS sources with two Chandra counterparts are listed here for completeness. XID numbers refer to Column 1 in the Cappelluti et al. 2009 catalog table: Nos. 35, 82, 131, 208, 215, 307, 336, 354, $365,380,384,419,2591,2618,5141,5208,5355,5556,10764,31163$, 53328, 54468, 60133, 60275, and 5210.

31 Publicly available at http://irsa.ipac.caltech.edu/data/COSMOS/tables/ib. The detection image, I-band mask, and SExtractor settings for the optical catalog were significantly modified from the version presented in Capak et al. (2007), which is now superseeded. All point-like sources brighter than 17th magnitude were modeled and subtracted from the detection image. This allowed the de-blending contrast to be set at a higher value, thus significantly decreasing the number of spurious sources without missing objects around bright sources and significantly reducing the number of close pairs blendings. In addition to lowering the contrast parameter, several of the detection parameters were adjusted to decrease the number of noise peaks at the faint end of the catalog. The combination of the change in background, de-blending, and detection results in small changes in the centroid and flux for some sources.
}

of spurious sources (mostly asteroids; Le Floc'h et al. 2009) and contains $\sim 50,000$ sources. In the XMM-COSMOS area, there are $\sim 36,000$ sources detected with an $\mathrm{S} / \mathrm{N}>5$ (implying a $24 \mu \mathrm{m}$ flux limit of $80 \mu \mathrm{Jy}$ ). We also matched the X-ray counterparts with the $70 \mu \mathrm{m}$ MIPS catalog (Frayer et al. 2009; Kartaltepe et al. 2010) which contains $\sim 1500$ sources down to $\sim 4 \mathrm{mJy}$, and with the VLA COSMOS catalog $(\sim 2400$ sources

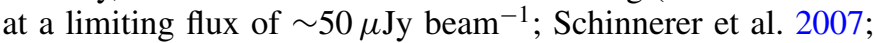
Bondi et al. 2008).

\section{IDENTIFICATION OF X-RAY SOURCES}

We have matched a counterpart to each of the 1797 X-ray sources as follows.

1. First, we associated the X-ray positions to the optical ones ( $I$ band) using the statistical method described in B07 (the "likelihood ratio technique;" Sutherland \& Saunders 1992; Ciliegi et al. 2003) in order to isolate the most obvious associations, and, at the same time, to pick up problematic cases (i.e., sources with two or more different counterparts with comparable likelihood or sources too faint to be identified). The method calculates the probability that a source is the correct association by weighting the information on the X-ray-to-optical distance, the surface density of (possible) false coincidence background objects and the brightness of the chosen counterpart (see B07 for other details). We divided the sources in three different classes: "reliable id," "ambiguous id" (comprising mostly two relatively bright sources with similar probability of being the correct optical counterpart), and "not identified" (comprising mostly faint or undetected sources for which the association is not statistically significant). Roughly, the 1797 sources were split into the three classes with the following percentages: $80 \%, 10 \%$, and $10 \%$, respectively, in agreement with the analysis reported in B07 that was limited to a subsample ( 700 sources, $40 \%$ ) of the complete XMM-COSMOS sample.

2. Then, we cross-correlated the optical positions with the $K$-band and IRAC catalogs. We created $I$-band (using Advanced Camera for Surveys (ACS) data in the inner $1.7 \mathrm{deg}^{2}$ area, the Subaru $I$-band data elsewhere), $K$-band, and IRAC $(3.6 \mu \mathrm{m}$ and $8.0 \mu \mathrm{m}) 20^{\prime \prime} \times 20^{\prime \prime}$ cutouts around each of the $1797 \mathrm{X}$-ray positions and visually checked the correctness of the optical/IR matches. Most of the "not identified" sources turned out to be associated to bright, isolated $K$ band or IRAC counterparts and therefore were moved into the "reliable id" sample. However, the diversity of the SED of objects in the sky led a large number of sources showing up in the IR, adding ambiguity to some of the proposed "reliable" associations. The distribution of the sources in the three classes after the correlation with the $K$-band and IRAC catalogs is 1458 sources $(81.3 \%)$ in the "reliable ID" class, 319 sources $(17.7 \%)$ in the "ambiguous ID" class, and 20 sources $(0.9 \%)$ in the "not identified" class.

\subsection{Chandra-XMM Matches and Position}

Chandra subarcsecond accurate X-ray positions are available for 850 of the 1797 XMM-COSMOS sources (see Section 2). Of these 850 objects, 712 were in the "reliable ID" class $(83.7 \%), 135(15.9 \%)$ were in the "ambiguous ID" class, and the remaining 3 were "not identified" $(0.4 \%)$. The lower percentage of not identified sources in the Chandra detected subsample can be due to the fact that many of the objects in this class might 

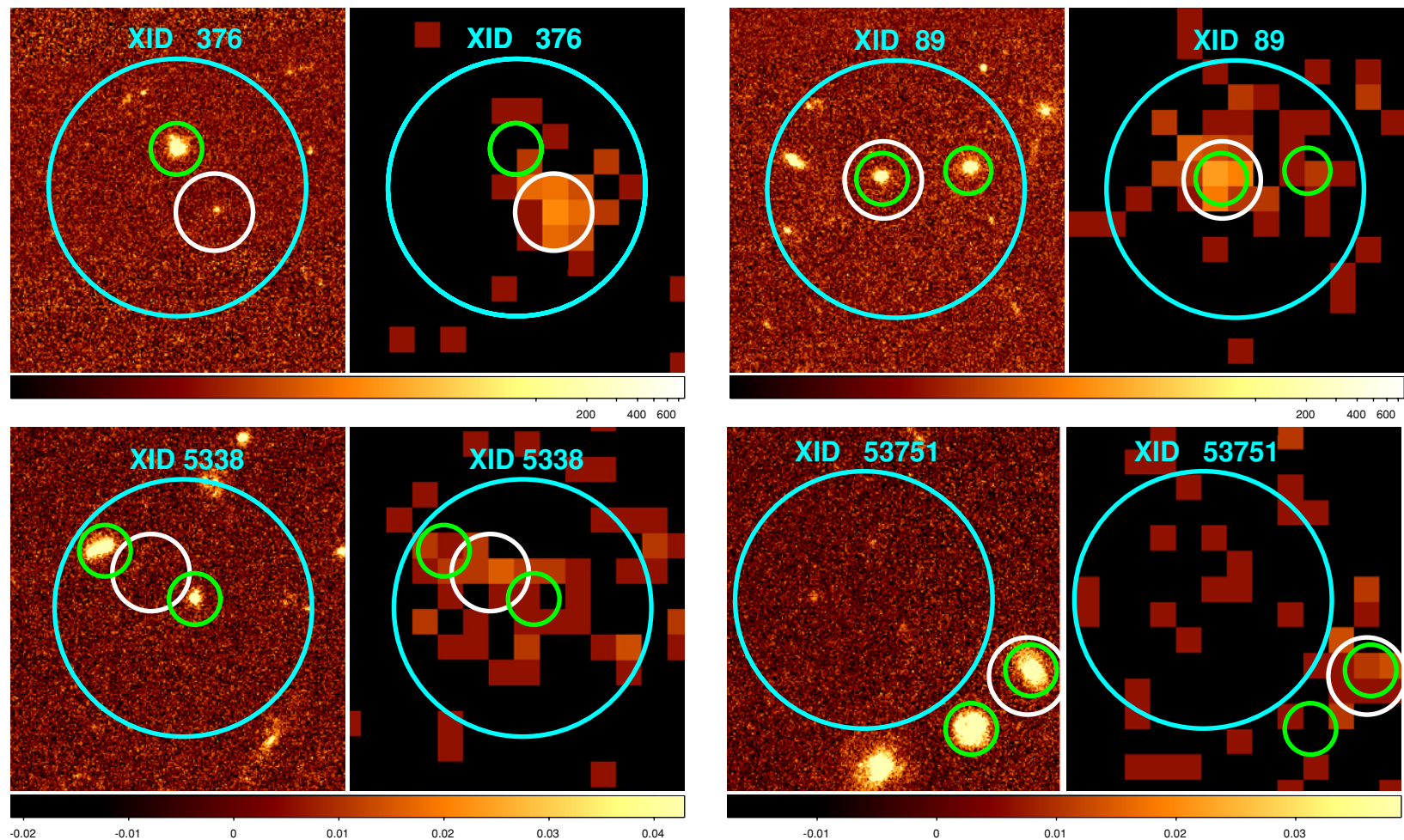

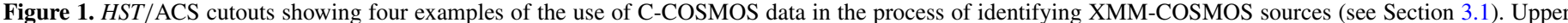

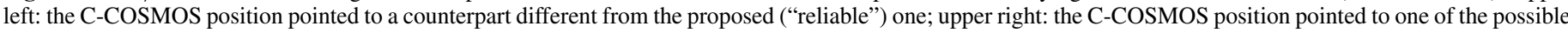

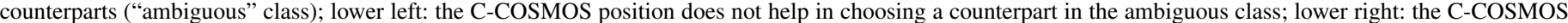

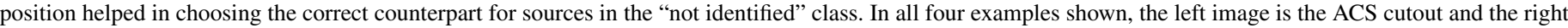

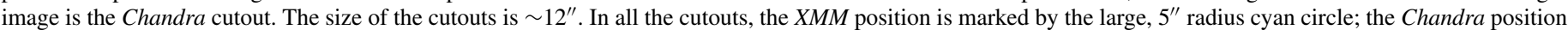

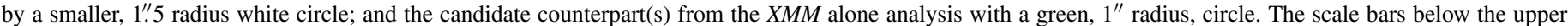

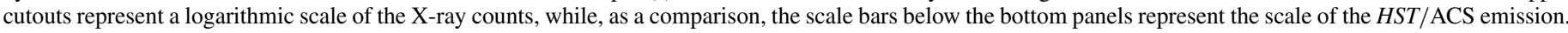
(A color version of this figure is available in the online journal.)

be spurious $X M M$ sources and therefore not even detected by Chandra (see also discussion in Elvis et al. 2009). Indeed, many of the sources without an optical id have a faint $X M M$ flux, close to the detection limit.

Of the 712 objects in the class of "reliable ID," Chandra pointed to a counterpart different from the one proposed in 12 cases (1.7\%; Figure 1, upper left panel). This fraction is lower for the brightest (flux limited, see below) sample (1.3\%) and rises up to $6 \%$ for the faintest sources. Among the 135 sources in the "ambiguous ID" class, in 114/135 cases (84.4\%) Chandra points to one of the two proposed associations (Figure 1, upper right panel), choosing the primary identifications in $50 \%$ of the cases and the secondary in the remaining half. For the remaining 21 objects $(15.6 \%)$ the Chandra information was not good enough to solve the ambiguity in the identification (Figure 1, lower left panel). Finally, all of the three "not identified" XMM sources were associated with a Chandra source at a large distance $\left(>5^{\prime \prime}\right)$ from the $X M M$ position and coincident with a relatively bright optical counterpart (Figure 1, lower right panel). We incorporated the Chandra information in our ID process and, at the end, we were able to reliably identify 1577 XMM sources $(87.7 \%)$; we still classify as "ambiguous ID" 203 sources $(11.3 \%)$ and we could not identify 17 sources (1.0\%). If we use the $1.7 \%$ of wrong identifications obtained above as an estimate of the failure rate of the likelihood ratio technique applied to the XMM-COSMOS data, we estimate that $\sim 12-13$ sources out of the 747 sources in the "reliable ID" class without Chandra coverage can still be associated with a wrong counterpart.

To maximize the completeness over a well-defined large area and, at the same time, keep selection effects under control, we further defined a subsample of sources detected above the limiting fluxes corresponding to a sky coverage of more than $1 \mathrm{deg}^{2}$ in at least one of the three $\mathrm{X}$-ray energy ranges considered, namely: $1 \times 10^{-15} \mathrm{erg} \mathrm{cm}^{-2} \mathrm{~s}^{-1}, 6 \times 10^{-15} \mathrm{erg} \mathrm{cm}^{-2} \mathrm{~s}^{-1}$, and $1 \times 10^{-14} \mathrm{erg} \mathrm{cm}^{-2} \mathrm{~s}^{-1}$, in the $0.5-2 \mathrm{keV}, 2-10 \mathrm{keV}$, or $5-10 \mathrm{keV}$ bands, respectively (see also Table 2 in C09). This subsample includes $1651 \mathrm{X}$-ray sources that will be used in the following to investigate the multiwavelength properties of $X M M$ counterparts (see Section 7). The combination of area and depth is similar to the one of the sample studied by Silverman et al. (2008, see their Figure 8) in the Chandra Multiwavelength Project survey (ChaMP; Kim et al. 2007); the main difference is given by the considerably higher-redshift completeness obtained in COSMOS thanks to the much deeper coverage in the optical and near-IR bands and the systematic use of photometric redshifts (see below). This drastically limits the need for substantial corrections for incompleteness (see discussion in Silverman et al. 2008). When imposing the limiting flux thresholds, the breakdown of the objects in the three classes is as follows: 1465 "reliable ID" (88.7\%), 175 "ambiguous" (10.6\%), and 11 $(0.7 \%)$ "not identified." A summary of the identification process and the breakdown in the classes of "reliable ID," "ambiguous ID," and "not ID," before and after the Chandra control-check, and before and after the inclusion of thresholds in X-ray fluxes is reported in Table 1 .

\section{THE XMM-COSMOS MULTIWAVELENGTH CATALOG}

The photometry at different wavelengths (except MIPS) has been cross-correlated with the positions of the XMM-COSMOS 
Table 1

Summary of Optical Identification of Point-like XMM Sources

\begin{tabular}{lcccc}
\hline \hline \multicolumn{1}{c}{ Sample } & Total Sources & Reliable $^{\mathrm{a}}(\%)$ & Ambiguous $^{\mathrm{b}}(\%)$ & Unidentified (\%) \\
\hline $\begin{array}{l}\text { Chandra } \text { area } \\
\text { (before } \text { Chandra } \text { check) }\end{array}$ & 850 & $712(83.8 \%)$ & $135(15.9 \%)$ & $3(0.3 \%)$ \\
$\begin{array}{l}\text { Chandra } \text { area } \\
\text { (after } \text { Chandra } \text { check) }\end{array}$ & 850 & $829(97.5 \%)$ & $21(2.5 \%)$ & $0(0 . \%)$ \\
\hline $\begin{array}{l}\text { XMM-COSMOS area } \\
\text { (before } \text { Chandra check) }\end{array}$ & 1797 & $1458(81 \%)$ & $319(18 \%)$ & $20(1.0 \%)$ \\
\hline $\begin{array}{l}\text { XMM-COSMOS area } \\
\text { (after } \text { Chandra check) }\end{array}$ & 1797 & $1577(87.7 \%)$ & $203(11.3 \%)$ & $17(1.0 \%)$ \\
\hline $\begin{array}{l}\text { XMM-COSMOS area } \\
\text { after flux thresholds) }\end{array}$ & 1651 & $1465(88.7 \%)$ & $175(10.6 \%)$ & $11(0.7 \%)$ \\
\hline
\end{tabular}

Notes.

a We classified as "reliable" those sources for which the multiwavelength analysis consistently point to the same counterpart, expected to be correct in $98 \%$ of the cases (see Section 3 for details).

b We classified as "ambiguous" those sources for which the multiwavelength analysis did not allow us to reliably associate a unique counterpart to the X-ray sources; for these objects, two sources with comparable likelihood are present in the $\mathrm{X}$-ray error box. See Section 3 for details.

counterparts (see Section 3) using a 0'.5 radius, and all the matches have been visually inspected, in order to remove false associations or include obvious matches at larger distances (> 0'.5). A significant number of sources $(\sim 10 \%)$ of the XMMCOSMOS counterparts turned out to be associated with blended objects in the IRAC catalog (e.g., the IRAC source is a clear blend of two different objects). Therefore, the photometry in the four different IRAC bands has been re-extracted at the position of the XMM-COSMOS counterparts, using a PSF fitting routine, allowing us a better determination of the fluxes for these blended IRAC sources. The MIPS catalog has been cross-correlated with the IRAC positions of the XMM-COSMOS counterparts using a radius of 2 .'5 and all the matches have been validated through a visual inspection. At the end, 1760 (98\%) sources in the XMM-COSMOS sample have associated entries from the "optical catalog," 1745 (97\%) have photometry from the " $K$-band catalog," $1711(95 \%)$ sources have IRAC $3.6 \mu \mathrm{m}$ photometry retrieved from the "IRAC catalog" or re-extracted at the position of the optical counterparts, and $1394(\sim 78 \%)$ have $24 \mu \mathrm{m}$ MIPS counterparts. Only two sources (XID 5120 and XID 556) have no associated photometry in any of the optical or infrared catalogs used. Both of them are close to bright stars $(I<16)$.

Table 2 lists the basic properties of the complete XMMCOSMOS counterpart sample: XMM-COSMOS IAU designation (Column 1); the XMM-COSMOS identifier number (from C09; Column 2); the coordinates of the optical/IR counterpart (J2000, Columns 3 and 4); the X-ray fluxes in the soft, hard, and ultra-hard bands (from C09; Columns 5, 6, and 7, respectively); a flag identifying the sources included in the flux-limited sample (Column 8); the X-ray hardness ratio, HR, defined as HR = $(H-S) /(H+S)$ where $H$ are the hard-band counts and $S$ the softband counts, respectively (Column 9); the Chandra-COSMOS identifier number (from Elvis et al. 2009; Column 10); the flag for the optical identification, according to the classes described in Table 1 (Column 11); the identifier number from Capak et al. (2007) catalog ${ }^{32}$ (Column 12); the identifier number as reported in the optical catalog and in Ilbert et al. (2009; Column 13);

\footnotetext{
32 The Capak et al. (2007) photometric catalog is available at IRSA, at the link: http://irsa.ipac.caltech.edu/data/COSMOS/tables/photometry/cosmos_phot_ 20060103.tbl.gz
}

the $r$-band and I-band magnitudes (from Capak et al. 2007; Columns 14 and 15); the $K$-band magnitude (from McCracken et al. 2010; Column 16); the magnitudes in the four IRAC channels (from Ilbert et al. 2009; Columns 17-20); the MIPS $24 \mu \mathrm{m}$ magnitude (from Le Floc'h et al. 2009; Column 21); the spectroscopic redshift (see Section 5; Column 22); the spectroscopic classification according to the classes outlined in Section 5.1 (Column 23); the origin of the spectroscopic redshift, with relevant notes (Column 24); the photometric redshift (from Salvato et al. 2009; Column 25). In case of ambiguous sources, all the possible counterparts of the XMM-COSMOS sources are listed. Table 2 is also available in ASCII format on the $\mathrm{Web}^{33}$.

The distribution of the X-ray-to-optical distances and of the I-band magnitudes for the XMM-COSMOS counterpart sample, derived considering the first counterpart listed in Table 2 for the 203 sources with an "ambiguous" counterpart, is very similar to those reported in B07. More specifically, about $90 \%$ of the reliable optical counterparts have an X-ray-to-optical separation $(\Delta(X-O))$ smaller than $3^{\prime \prime}$ which is better than the $\sim 80 \%$ found in XMM-Newton data of comparable depth (see, e.g., Fiore et al. 2003, hereinafter F03; Della Ceca et al. 2004; Loaring et al. 2005). The improvement is likely given by the combination of the accurate astrometry of the positions of the X-ray sources (tested with simulations in Cappelluti et al. 2007), the full exploitation of the multiwavelength catalog (optical and infrared) for the identification process, and the availability of Chandra accurate positions. The majority of the XMM-COSMOS counterparts $(\sim 68 \%)$ have optical $\left(I_{\mathrm{AB}}\right)$ magnitudes in the range $20<I_{\mathrm{AB}}<24$, with a median magnitude of $I_{\mathrm{AB}}=21.98$ (with dispersion of 1.35), and two symmetric tails at fainter and brighter magnitudes. The median magnitude of optical counterparts is slightly fainter than, but still consistent with, the median value presented by B07. The above mentioned distributions do not change if we make a different choice for the optical counterpart of the 203 "ambiguous" sources, i.e., the second most likely optical counterpart is considered instead. As an example, the median optical magnitude would be $I_{\mathrm{AB}}=21.96$ (with dispersion 1.36). The same applies to other quantities (e.g., $K$-band magnitudes, photometric redshifts etc.). Therefore, from a statistical point of

\footnotetext{
33 http://www.mpe.mpg.de/XMMCosmos/xmm53_release/
} 
Table 2

Basic Properties of the XMM-COSMOS Counterparts ${ }^{\mathrm{a}}$

\begin{tabular}{|c|c|c|c|c|c|c|c|c|c|c|c|c|c|c|c|c|c|c|c|c|c|c|c|c|}
\hline IAU Name & XID & $\begin{array}{l}\text { R.A. } \\
(\mathrm{deg})\end{array}$ & $\begin{array}{l}\text { Decl. } \\
(\mathrm{deg})\end{array}$ & $\begin{array}{c}S_{0.5-2} \\
1 \mathrm{e}-13 \mathrm{cgs} \\
\end{array}$ & $\begin{array}{c}S_{2-10} \\
1 \mathrm{e}-13 \mathrm{cgs} \\
\end{array}$ & $\begin{array}{c}S_{5-10} \\
1 \mathrm{e}-13 \mathrm{cgs}\end{array}$ & Flag & $\mathrm{R}$ & ID & FlagID & k) & t) & $\begin{array}{c}r \\
\mathrm{AB} \\
\end{array}$ & $\begin{array}{c}I \\
\mathrm{AB} \\
\end{array}$ & $\begin{array}{c}K \\
\mathrm{AB} \\
\end{array}$ & $\begin{array}{c}3.6 \mu \mathrm{m} \\
\mathrm{AB} \\
\end{array}$ & $\begin{array}{c}4.5 \mu \mathrm{m} \\
\mathrm{AB} \\
\end{array}$ & $\begin{array}{c}5.8 \mu \mathrm{m} \\
\mathrm{AB}\end{array}$ & $\begin{array}{c}8.0 \mu \mathrm{m} \\
\mathrm{AB}\end{array}$ & $\begin{array}{c}24 \mu \mathrm{m} \\
\mathrm{AB} \\
\end{array}$ & zspec & Class & Notes & zphot \\
\hline 82 & 1 & 50.10521 & 981183 & 13900 & 22800 & 12200 & 1 & 50 & & 1 & & & 19.24 & 19.12 & 18.21 & 17.49 & 17.20 & 16.89 & 16.67 & 15.10 & 0.373 & 1 & & $\overline{0.37}$ \\
\hline 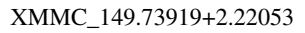 & 2 & & & & & & 1 & & & 1 & & & & 19.96 & & & & & & & & 1 & & .05 \\
\hline & 3 & & & & & & & & & & & & & 1 & & & & & & 48 & & & & 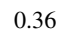 \\
\hline & 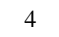 & & & & & & & & & & & & & 1 & & & & & & & & & & .11 \\
\hline & 5 & & & & & & 1 & & & & & & 19.90 & 19.47 & - & & & & & 93 & 57 & 1 & & 18 \\
\hline & 6 & & & & & & & & & & & & & & & & & & & & & & & 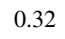 \\
\hline & 7 & & & & & & & & - & & & & 5 & 4 & & & & 3 & 6 & 3 & 9 & & & 1.36 \\
\hline 967 & 8 & 05378 & 89671 & & & & 1 & & 142 & 1 & & & 19.29 & 18.79 & 18.20 & 22 & 16.89 & 53 & 16.24 & 15.07 & 0.699 & 1 & 1 & 0.71 \\
\hline & 0 & & & & & & & & 4 & & & & 20 & 20.07 & . & & 18.36 & 38 & 41 & 16.05 & & & 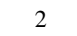 & 1.46 \\
\hline MMC_149.91261+2.20032 & 10 & 49.91244 & 00366 & 7.20 & 2.10 & 0.90 & 1 & 0.72 & 446 & 1 & 1660949 & 58 & 20.88 & 20.39 & 19.37 & 18.72 & 18.50 & 18.22 & 17.93 & 15.74 & 0.689 & 1 & 2 & 0.68 \\
\hline
\end{tabular}

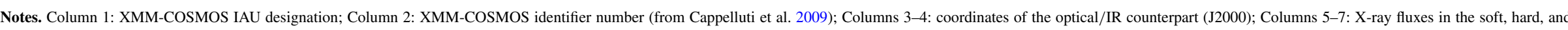

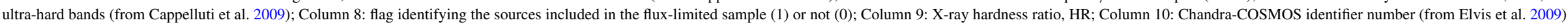

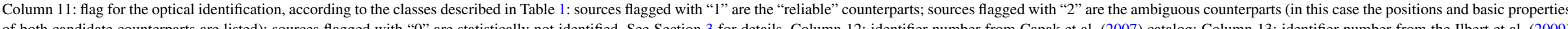

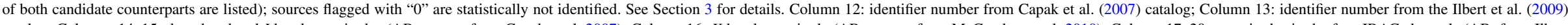

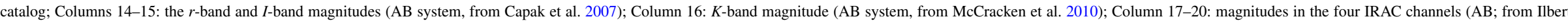

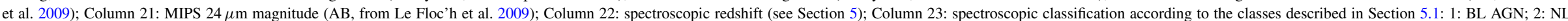

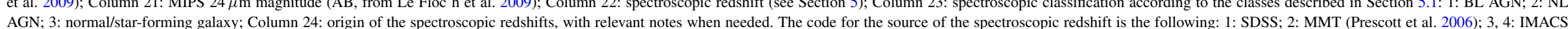
AGN; 3: normal/star-forming galaxy; Column 24: origin of the spectroscopic redshifts, with relevant notes when needed. The code for the source of the spectroscopic redshift is the follow
runs (Trump et al. 2007, 2009); 5: zCOSMOS 20k catalog (Lilly et al. 2007); 6: zCOSMOS faint 4.5k catalog; 7; Keck runs; Column 25: photometric redshift (from Salvato et al. 2009).

runs (Trump et al. 2007, 2009); 5: zCOSMOS 20k catalog (Lilly et al. 2007); 6: zCOSMOS faint 4.5k catalog; 7; Keck runs; Colu
a The information on the possible second counterpart for the sources in the "ambiguous" ID class are listed at the end of the table.

(This table is available in its entirety in a machine-readable form in the online journal. A portion is shown here for guidance regarding its form and content.) 

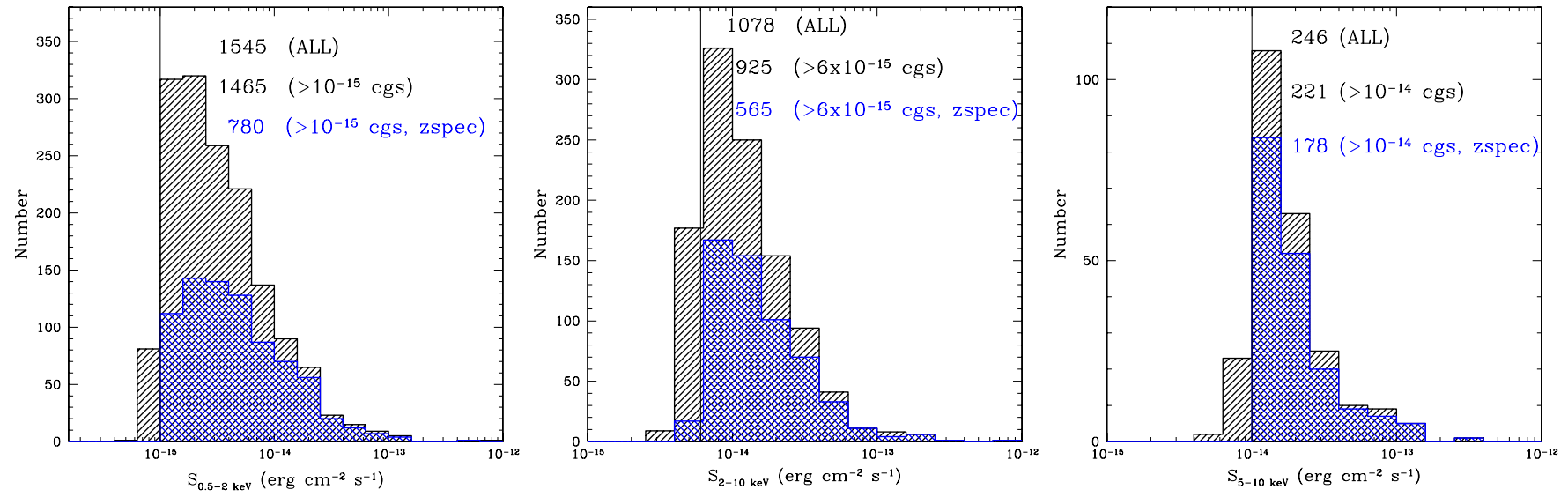

Figure 2. Histograms of the flux distributions for the soft (0.5-2 keV, 1545 sources; left), hard (2-10 keV, 1078 sources; middle), and ultra-hard (5-10 keV, 246 sources; right) samples. The vertical line shows the flux at which the sky coverage is $1 \mathrm{deg}^{2}$ (with 1465,925 , and $221 \mathrm{soft}$, hard, and ultra-hard sources, respectively). The blue histograms show the flux distributions for the subsamples of sources with spectroscopic redshift, including 780, 565 , and 178 sources in the soft, hard, and ultra-hard band, at fluxes higher than the adopted thresholds, respectively.

(A color version of this figure is available in the online journal.)

view, the multiwavelength properties discussed in this paper can be considered representative of the overall X-ray point source population, at the sampled X-ray fluxes.

\section{SPECTROSCOPIC AND PHOTOMETRIC REDSHIFTS AND SOURCE BREAKDOWN}

Good-quality spectroscopic redshifts for the proposed counterparts are available from Magellan/IMACS and MMT observation campaigns ( $\sim 530$ objects; Trump et al. 2007, 2009), from the VIMOS/zCOSMOS bright project ( $\sim 500$ objects; Lilly et al. 2009), from the VIMOS/zCOSMOS faint project ( $\sim 80$ objects; Lilly et al. 2007$)$ or were already present either in the Sloan Digital Sky Survey (SDSS) catalog ( $\sim 100$ objects; Adelman-McCarthy et al. 2006; Kauffmann et al. $2003^{34}$ ) or in the literature ( $\sim 95$ objects; Prescott et al. 2006). Additional $\sim 40$ objects have redshifts from ongoing spectroscopic campaigns designed to target high-redshift, faint objects with the DEIMOS instrument at Keck-II telescope (PI: P. Capak, M. Salvato, N. Scoville). In summary, a total of 890 unique, good-quality spectroscopic redshifts are available for XMM-COSMOS sources, 852 when the XMM-COSMOS flux-limited sample is considered, corresponding to a substantial fraction $(\sim 52 \%$, $852 / 1651)$ of the sample investigated in this paper.

Figure 2 shows the flux distributions in the soft, hard, and ultra-hard bands, respectively, for the entire X-ray source population (black histograms) and for the subsample with spectroscopic identifications (blue histograms). The vertical line shows the flux at which the sky coverage is $1 \mathrm{deg}^{2}$ (with 1465, 925 , and 221 soft, hard, and ultra-hard sources, respectively). The spectroscopic completeness increases with the energy band considered: it is 53\% (780/1465) in the soft band, 61\% (565/ $925)$ in the hard band, while the highest percentage is reached in the ultra-hard band $(178 / 221, \sim 80 \%)$. The high spectroscopic completeness in the ultra-hard sample reflects the fact that the sources are detected at bright fluxes and, therefore, associated on average to bright optical counterparts (median $I_{\mathrm{AB}} \sim 20.7$, to be compared to the median magnitude of the entire sample, $I_{\mathrm{AB}} \sim 22$; see Section 4) therefore favoring the spectroscopic follow-up.

\footnotetext{
34 These sources have been retrieved from the NED, NASA Extragalactic Database and from the SDSS archive.
}

Photometric redshifts for all the XMM-COSMOS sources have been obtained exploiting the COSMOS multiwavelength database and are presented in Salvato et al. (2009). Since the large majority of the XMM-COSMOS sources are AGNs, in addition to the standard photometric redshift treatments for normal galaxies, a new set of SED templates has been adopted, together with a correction for long-term variability and luminosity priors for point-like sources (see Salvato et al. 2009 for further details). Thanks also to the availability of the intermediate band Subaru filters (Taniguchi et al. 2007), crucial in picking up emission lines (see also Wolf et al. 2004), we were able, for the first time for an AGN sample, to obtain photometric redshift accuracy comparable to that achieved for inactive galaxies $\left(\sigma_{\Delta z /(1+z)} \sim 0.015\right.$ and $\sim 5 \%$ outliers $)$ at $I \lesssim 22.5$. At fainter magnitudes $(22.5<I<24.5)$ the dispersion increases to $\sigma_{\Delta z /(1+z)} \simeq 0.023$ with $\sim 10 \%$ outliers, still remarkably good for an AGN sample. A photometric redshift is available for all but 31 (24) objects out of 1797 (1651) objects in the complete (flux-limited) XMM-COSMOS counterpart sample. About half of them do not have wide multiband photometry, being detected only in the IRAC and $K$ bands. The remaining half are affected by severe blending problems in the IR making the photo- $z$ estimate unreliable.

Considering the spectroscopic and photometric sample, we have an almost $100 \%$ completeness in redshift for the XMMCOSMOS sources: only 28 sources do not have a photometric or spectroscopic measured redshift (21, i.e., less than $1.3 \%$, in the flux-limited sample).

The left panel of Figure 3 shows, as an open histogram, the redshift distribution of the objects in the XMM-COSMOS counterpart sample. Only extragalactic sources are considered here. The black solid histogram shows the distribution of the objects with available spectroscopic redshifts. The redshift distribution shows prominent peaks at various redshifts $(z \sim$ $0.12, z \sim 0.36, z \sim 0.95, z \sim 1.2, z \sim 2.1$ ), both in the spectroscopic and photometric subsamples. In particular, the structure at $z \sim 0.36$ is the one with the highest overdensity with respect to a smooth distribution, being present over many wavelengths in the COSMOS field (see Lilly et al. 2009), and is likely responsible for the excess signal observed in the clustering amplitude of X-ray sources around $r_{p} \sim 5-15 h^{-1}$ Mpc described in details in Gilli et al. (2009). 

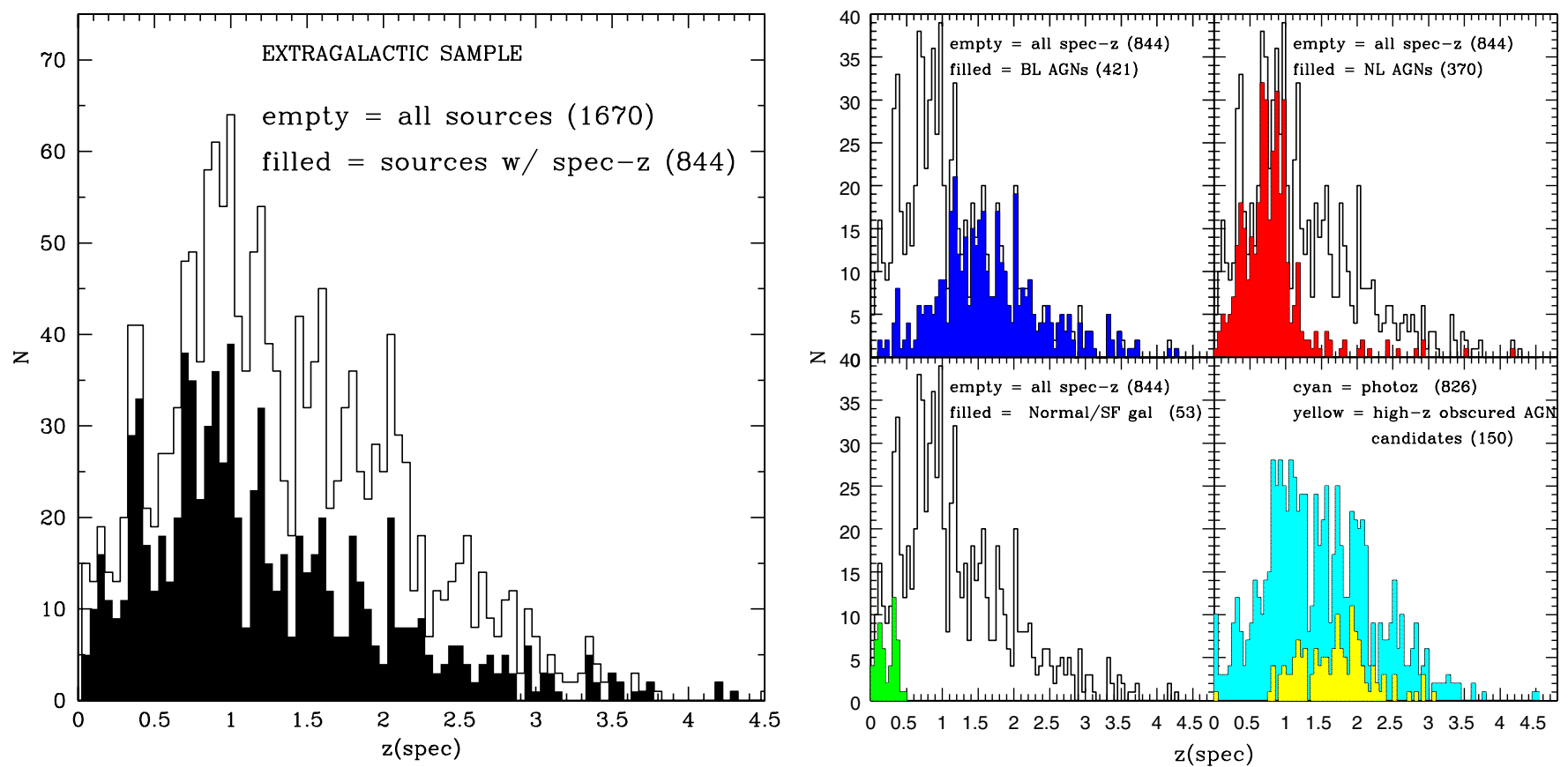

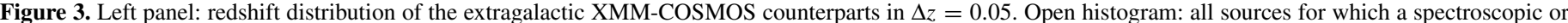

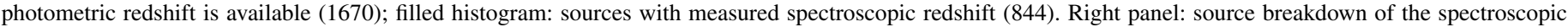

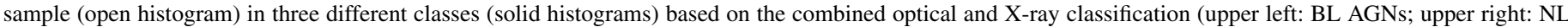

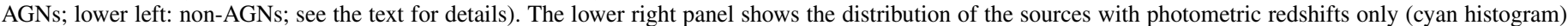
and, as a comparison, the redshift distribution for the high- $z$ obscured AGN candidates discussed in Section 7 . The redshift bin in all the panels is $\Delta z=0.05$.

(A color version of this figure is available in the online journal.)

The XMM-COSMOS counterpart sample has 46 spectroscopically confirmed galactic stars. Additional 53 objects are classified as stars on the basis of the inspection of the optical finding charts and/or the observed SED, for which the best-fit solution is obtained with a stellar template (see Salvato et al. 2009). Overall, 99 objects are classified as stars, making up the $5.5 \%$ of the entire XMM-COSMOS counterpart sample $(6.5 \%$ when the soft sample is considered), in agreement with results from other large area X-ray surveys at similar limiting fluxes (e.g., Covey et al. 2008 from the Champ Extended Stellar Survey, Chess). We refer to a paper in preparation (B. Stelzer et al. 2010 , in preparation) for a detailed analysis of the stars contents in XMM-COSMOS.

\subsection{Spectroscopic Breakdown}

For the purposes of the present paper, we divided the extragalactic sources with available optical spectra in the XMMCOSMOS counterpart sample into three classes, on the basis of a combined X-ray and optical classification (see also Szokoly et al. 2004). A more detailed analysis of the optical spectra will be presented in future papers, based also on more complete data that are rapidly being accumulated from ongoing projects.

1. Broad-line $A G N$ (BL $A G N$ or $A G N 1$ hereafter). All the objects having at least one broad (FWHM $>2000 \mathrm{~km} \mathrm{~s}^{-1}$ ) optical emission line in the available spectrum (421 sources, 416 in the flux-limited sample).

2. Non-broad-line AGN (NL AGN or AGN 2 hereafter). All the objects with unresolved, high-ionization emission lines, exhibiting line ratios indicating AGN activity, and, when high-ionization lines are not detected, or the observed spectral range does not allow to construct line diagnostics, objects without broad line in the optical spectra and with rest-frame hard X-ray luminosity in excess than $2 \times$
$10^{42} \mathrm{erg} \mathrm{s}^{-1}$ typical of AGNs (370 sources, 344 in the flux-limited sample; about 1/3 from line diagnostics; see below for details).

3. "Normal" galaxies. All the objects with unresolved emission lines consistent with spectra of star-forming galaxies or with a typical galaxy spectrum showing only absorption lines, and with rest-frame hard X-ray luminosity smaller than $2 \times 10^{42} \mathrm{erg} \mathrm{s}^{-1}$, or undetected in the hard band (53 sources, 49 in the flux-limited sample).

The choice of using a combination of X-ray and optical criteria in the classification was motivated by the fact that both obscured and unobscured AGNs can be misclassified in spectroscopic studies, given that the host-galaxy light may overshine the nuclear emission (as in the cases of the XBONG; Moran et al. 2002; Comastri et al. 2002; Severgnini et al. 2003; Civano et al. 2007). Moreover, at high redshift $(z>0.5)$, the observed wavelength range does not allow one to construct the standard line ratio diagnostics ("BPT" diagrams; Baldwin et al. 1981; Kewley et al. 2001) used to distinguish between star-forming and Type 2 AGNs at lower redshifts $(z<0.5)$, where a more accurate classification is usually possible (see, e.g., discussion in Bongiorno et al. 2010 for the zCOSMOS Type 2 AGN sample). We chose a luminosity threshold of 2 $\times 10^{42} \mathrm{erg} \mathrm{s}^{-1}$ in the hard band ${ }^{35}$ to discriminate between star formation processes and accretion processes as responsible for the X-ray emission, following two arguments: (1) the most powerful local, starburst galaxy, NGC 3256 has a $2-10 \mathrm{keV}$ luminosity lower than this value $\left(\sim 5 \times 10^{41} \mathrm{erg} \mathrm{s}^{-1}\right)$ and (2) following Ranalli et al. (2003), this luminosity level can be due to stellar processes only in objects with star formation rate (SFR)

\footnotetext{
35 When a source is detected only in the soft band the corresponding luminosity threshold used is $10^{42} \mathrm{erg} \mathrm{s}^{-1}$.
} 


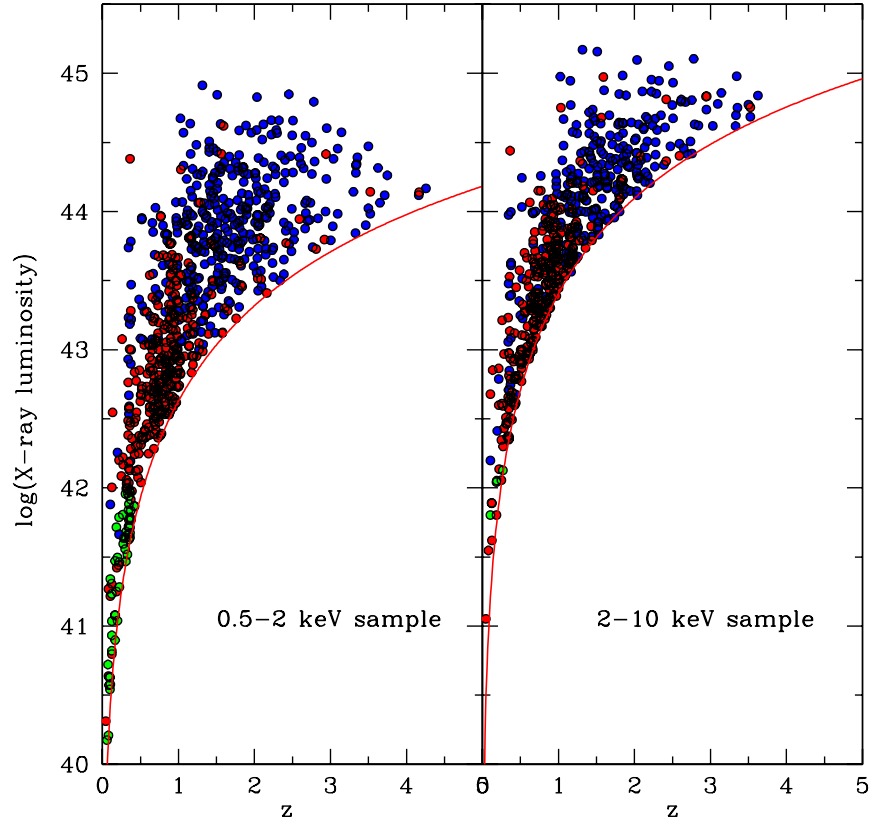

Figure 4. Left panel: luminosity-redshift plane for the sources with spectroscopic redshift detected in the soft band. Blue circles are AGN 1s, red circles are AGN 2s, and green circles are "normal" galaxies (see Section 5.1 for the definition). The continuous line represents the completeness limit in the sample. Right panel: the same as left panel, for the hard-band sample.

(A color version of this figure is available in the online journal.)

larger than $\sim 400 M_{\odot} \mathrm{yr}^{-1}$ (> $2000 M_{\odot} \mathrm{yr}^{-1}$ for luminosities larger than $10^{43} \mathrm{erg} \mathrm{s}^{-1}$, typical of the large majority of our objects). Furthermore, given the flux sensitivity of the XMMCOSMOS survey (solid line in Figure 4), objects less luminous than $2 \times 10^{42} \mathrm{erg} \mathrm{s}^{-1}$ cannot be detected at redshift $z \gtrsim 0.5$ (see also the next section), where optical spectroscopic classification starts to become problematic.

The right panel of Figure 3 shows the spectroscopic breakdown of the extragalactic sample in the three classes reported above (upper left, upper right, and lower left quadrants, respectively). We also show the redshift distribution of the sources for which only photometric redshifts are available (lower right quadrant). Among the spectroscopically identified sample, BL AGNs make up $\gtrsim 50 \%$ in both the soft and hard subsamples, and more than $60 \%$ in the ultra-hard sample. The higher fraction of BL AGN in the ultra-hard sample is mainly due to the shallower sensitivity of $X M M$ at energies $>5 \mathrm{keV}$, which limits the detection to the brightest flux/luminosities where the fraction of obscured AGNs is lower (see also Section 8). The contribution of non-AGN sources (normal galaxies and stars) is maximum in the soft sample $(\sim 12 \%, 97$ objects are classified in these two classes), decreases to $\sim 3 \%$ in the hard band ( 16 objects classified as stars or non-AGNs) and only two sources are classified in these two classes in the ultra-hard band.

Figure 4 shows the luminosity-redshift plane for the sources with spectroscopic redshift detected in the soft (left panel) and in the hard band (right panel), respectively. Different colors refer to different AGNs or galaxy classes as detailed above. Among the sources in the XMM-COSMOS sample with spectroscopic redshifts, only 64 (12) of the objects classified as NL AGNs are located at $z>1(z>2)$, to be compared with 350 (124) in the BL AGN sample. This is mostly due to the fact that high-redshift NL AGNs are optically faint (typically $I \sim 23-24$ ) and have not been targeted yet with dedicated spectroscopic campaigns (see also Eckart et al. 2006; Caccianiga et al. 2008; Stalin et al. 2010).

\section{NUMBER DENSITY EVOLUTION}

The high completeness in optical identifications of the XMMCOSMOS sample and the availability of spectroscopic and photometric redshifts allow us to estimate the number densities of AGNs as a function of luminosity and redshift. A discussion of the X-ray luminosity function is beyond the scope of this paper; a comprehensive analysis using XMM-COSMOS and C-COSMOS data, combined with archival data, will be the subject of a future paper (T. Miyaji et al. 2010, in preparation). In this section, we compare our first estimates of the number density evolution of the most luminous AGN population, for which XMM-COSMOS can be considered reasonably complete, with other recent measurements. In this analysis, we use the traditional $\sum 1 / V_{\mathrm{a}}$ estimator (Avni \& Bahcall 1980).

In calculating the binned number density evolution, we considered only the subsample of sources detected in the hard (2-10 keV) band and included in the flux-limited sample (925 sources at $S_{\mathrm{x}}=6 \times 10^{-15} \mathrm{erg} \mathrm{s}^{-1} \mathrm{~cm}^{-2}$; see Section 5 and Figure 2). We limited the analysis to sources that have ID-class "reliable" or "ambiguous" (Table 1). As discussed in Section 4, the statistical properties of the "primary" counterparts of those in the "ambiguous" class are very similar to those of the secondary objects in the same class. Thus, we use the primary identifications for the "ambiguous" class objects. In calculating the available volume $V_{\mathrm{a}}$, we have used the curve of the hard-band survey solid angle versus flux limit from C09, calibrated with extensive simulations and reproducing the observed $\log N-\log S$ distribution (Cappelluti et al. 2007; C09).

Using the HR between the soft- and hard-band count rates and the $X M M$-EPIC energy response functions, we have estimated the absorption column density $\left(N_{\mathrm{H}}\right)$ of each source. If the AGN was not detected in the soft band, the upper limit was used instead. In calculating $N_{\mathrm{H}}$, we have assumed a $\Gamma=1.8$ power law for the unabsorbed spectrum. If the HR were softer than the $\Gamma=1.8$ intrinsic power law, we have set $N_{\mathrm{H}}=0$ and used the $\Gamma$ calculated from the HR. The derived $N_{\mathrm{H}}$ values are in good agreement with those of the full X-ray spectroscopic analysis of bright XMM-Newton sources in the sample (Mainieri et al. 2007; V. Mainieri et al. 2010, in preparation). The $N_{\mathrm{H}}$ estimates have been used to derive de-absorbed X-ray luminosities.

The available volumes $V_{\mathrm{a}}$ have been calculated with $K$-corrections assuming the above spectrum, folded with the hard-band response curve of the XMM-Newton EPIC detectors $(\mathrm{pn}+2 \mathrm{MOS})$. Binning in luminosity has been made using the de-absorbed luminosities in the rest-frame $2-10 \mathrm{keV}$. The sizes of the redshift bins have been determined adaptively such that there are 20 objects in each bin. If the 20 objects bin is smaller than $\Delta z=0.2$ or larger than 0.9 , these minimum and maximum sizes are used. The last two redshift bins have been adjusted manually such that they contain approximately the same number of objects.

The results are presented in Figure 5, where the number density of XMM-COSMOS sources with $\log L_{X}=44.5-47 \mathrm{erg} \mathrm{s}^{-1}$ is plotted versus the redshift (black squares). In the calculation of the space density, we limited the redshift interval to $z=3$, where the $L_{X}$ corresponds to a $2-10 \mathrm{keV}$ flux above our limit (see right panel of Figure 4). In the same figure, we plot the data points obtained from the AEGIS survey by Aird et al. (2010; black circles at $z<1.2$ red circles at $z>2$ ), along with their best-fit luminosity function (solid black curve). We 


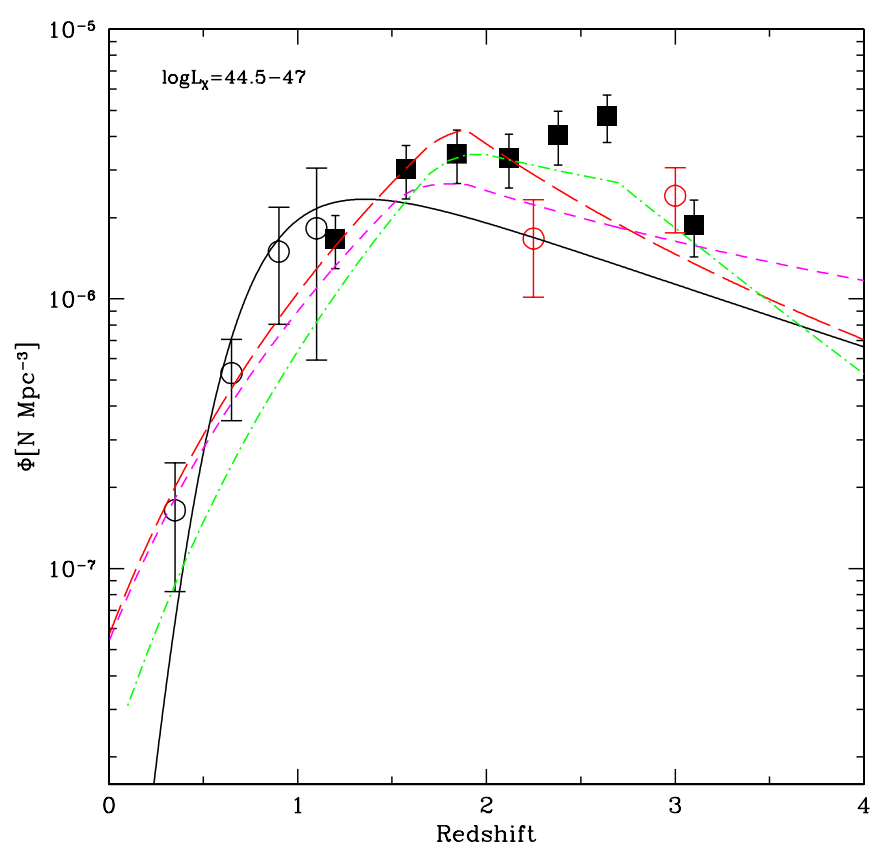

Figure 5. Number density evolution from the XMM-COSMOS AGNs in the luminosity range $\log L_{X}=44.5-47$ and in the redshift range $z=1-3$ (black squares), compared with recent results from Aird et al. (2010; open circles/ black solid lines), Ebrero et al. (2009; pink dot-dashed curves), and Silverman et al. (2008; red dotted curves). The green dot-long-dashed curve represents the expectation of XRB synthesis models from Gilli et al. (2007).

(A color version of this figure is available in the online journal.)

also plot the number density evolution for the XLF models from Ebrero et al. (2009; pink dot-dashed curve), and Silverman et al. (2008; red dotted curve), and the expectations from $\mathrm{X}$-ray background (XRB) synthesis models (Gilli et al. 2007; green dot-long-dashed curve). The XMM-COSMOS and Aird et al. (2010) data points at $z>2$ are consistent within $\sim 2 \sigma$ and the difference between the models can be ascribed to the way in which the models are fit to the data. The XMM-COSMOS data points seem to favor a higher-redshift $(z \sim 2)$ peak for the space density of luminous quasars, more consistent with an LDDE parameterization (represented by the Ebrero et al. 2009 and Silverman et al. 2008 curves, though different in the details; see also Yencho et al. 2009), than with the lower-redshift peak expected from the LADE model recently proposed by Aird et al. (2010), which is a factor of 2.5 lower than our points at $z \sim 2.5$. In particular, the number density evolution reproduced by XRB synthesis models (Gilli et al. 2007) accounts for both the COSMOS and the Aird et al. (2010) points to within the error bars.

\section{LUMINOUS OBSCURED AGNs}

In this section, we discuss the strategy adopted to build a sample of luminous ( $\log L_{X}>44 \mathrm{erg} \mathrm{s}^{-1}$ ) obscured AGNs at moderate to high redshifts $(1 \lesssim z \lesssim 2.5)$, fully exploiting the photometric and spectroscopic information presented in the previous sections. In particular, we make use of the high spectroscopic redshift completeness at relatively bright fluxes, and the availability of spectral classifications, to assess the reliability/robustness of color-color diagrams and flux ratios as diagnostics of the presence of an obscured AGN, i.e., objects for which the optical nuclear emission is blocked (no broad lines in the optical spectra) and/or characterized by substantial $\left(\log N_{\mathrm{H}} \gtrsim 21.5\right) \mathrm{X}$-ray obscuration. We will then apply these diagnostics to select and study the fainter sources for which only photometric redshifts are available. The devised method may complement systematic studies based on SED fitting, allowing one to efficiently isolate luminous, obscured AGNs at high- $z$ for more detailed follow-up analyses. All the statistical properties, correlation analyses and figures will be presented for the flux-limited sample of 1651 sources, since it is less affected by incompleteness, and we expect a lower fraction (1.3\%) of wrong identifications (see Section 3.1). We further exclude from the analysis the 11 sources in this sample for which we are not able to provide a reliable identification from our multiwavelength analysis (see Sections 3 and 3.1). The multiwavelength properties of fainter X-ray detected AGNs $\left(F_{0.5-2 \mathrm{KeV}} \lesssim 10^{-15} \mathrm{erg} \mathrm{cm}^{-2} \mathrm{~s}^{-1}\right)$ will be investigated in the framework of the C-COSMOS survey (F. Civano et al. 2010, in preparation).

\subsection{Selection and Sample Construction}

A proper source classification (AGN versus starburst versus passive galaxy) should ideally be obtained via a complete analysis of its emission over the entire electromagnetic spectrum, using both spectroscopic (e.g., emission line widths and ratios, see, e.g., Bongiorno et al. 2010) and photometric (SED; Lusso et al. 2010; M. Elvis et al. 2010, in preparation) observables. However, a complete source characterization is difficult to obtain even in fields where the best and deepest imaging and spectroscopic campaigns have been obtained, such as the COSMOS field. A reliable information (at least in a statistical sense) can be obtained through the analysis of the emission in bands where differences between nuclear and star formation emission are emphasized. In particular, the combination of observed-frame mid-infrared, near-infrared, and X-ray-tooptical flux ratios has been exploited recently in the literature to isolate obscured AGNs (see, e.g., F03; Martinez-Sansigre et al. 2005, 2006; Fiore et al. 2008; Donley et al. 2008; Dey et al. 2008). We will apply in the following several multiwavelength diagnostics in order to study the properties of the most obscured sources in the XMM-COSMOS sample.

\subsubsection{X-ray-to-Optical and Optical to Near-infrared Colors $(X / O$ and $R-K)$}

Since the first source identification campaigns of hard $\mathrm{X}$-rays surveys, a class of sources with high $(>10) \mathrm{X}$ ray-to-optical flux ratio $\left(\mathrm{X} / \mathrm{O}^{36}\right.$; Hornschemeier et al. 2001; Alexander et al. 2001; Giacconi et al. 2001; F03; Koekemoer et al. 2004; Eckart et al. 2006; Cocchia et al. 2007) has been found. This value of $\mathrm{X} / \mathrm{O}$ is significantly higher than the average value observed for optically and soft X-ray selected AGNs (Maccaccaro et al. 1988). Later studies (e.g., Perola et al. 2004; Civano et al. 2005) found that the high$\mathrm{X} / \mathrm{O}$ sources tend also to be obscured in the X-rays, with column densities of the order of $10^{22}-10^{23} \mathrm{~cm}^{-2}$, and that sources with $\mathrm{X} / \mathrm{O}>10$ selected at bright fluxes $\left(F_{2-10} \gtrsim 10^{-14} \mathrm{erg} \mathrm{cm}^{-2} \mathrm{~s}^{-1}\right)$ are candidate high-luminosity obscured quasars. Also, these sources are detected preferentially at $z>1$ and characterized on average by red optical to near-infrared colors $(R-K \gtrsim 5$, in the Vega system) pointing toward a strong link between X-ray detected extremely red objects and Type 2 Quasars (Alexander

\footnotetext{
${ }^{36}$ We define $\mathrm{X} / \mathrm{O}=f_{2-10 \mathrm{keV}} / f_{r \text {-band }}$, where the $R$-band flux is computed by converting $r$-band magnitudes into monochromatic fluxes and then multiplying them by the width of the $r$-band filter (Zombeck 1990).
} 


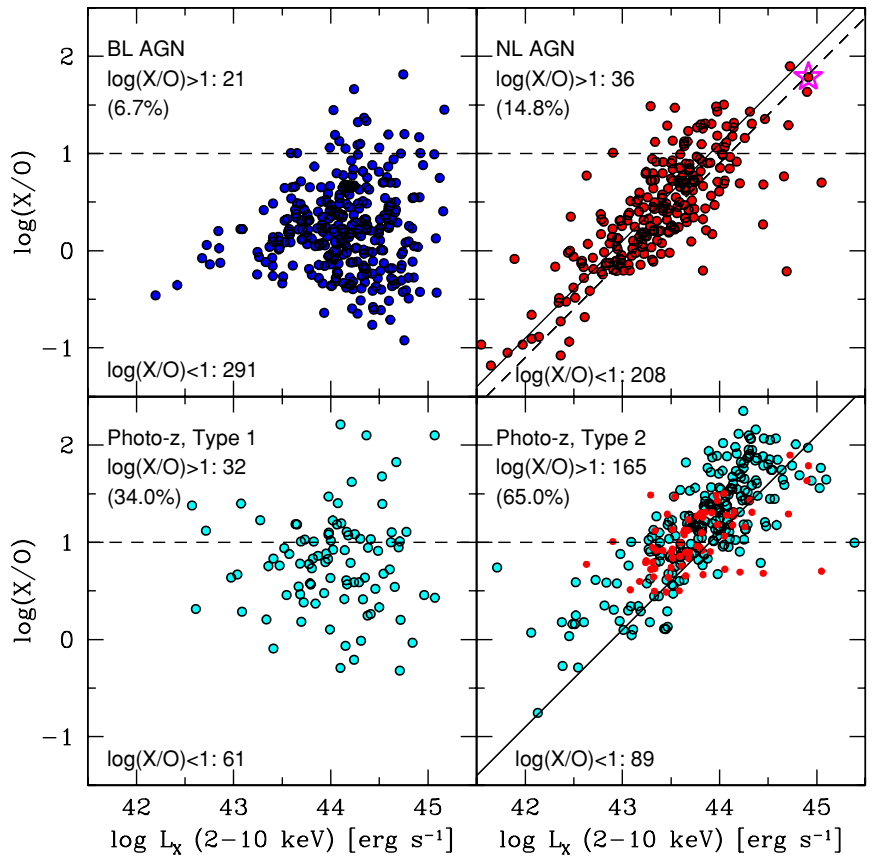

Figure 6. Upper panels: $\mathrm{X} / \mathrm{O}$ vs. the de-absorbed rest-frame hard X-ray luminosity for the sources classified as BL AGNs (left, blue circles) and for the sources classified as AGN 2s (right, red circles). The dashed diagonal line marks the F03 relation between the $\mathrm{X} / \mathrm{O}$ and the $2-10 \mathrm{keV}$ luminosity for obscured AGNs. The solid line marks the best-fit relation obtained for the XMM-COSMOS sample (see the text for details). The magenta star marks the position in this diagram of XID 2028 (see Section 8). Lower panels: X/O vs. the rest-frame hard X-ray luminosity for the sources with photometric redshifts and classified from the analysis of the spectral energy distribution as AGN dominated (left) and as galaxies dominated (right). As red circles, we also plot the spectroscopically identified AGN 2s (taken from the upper panel) with faint optical magnitudes $(R>23)$. The solid line marks the best-fit relation obtained for the XMM-COSMOS spectroscopic sample of NL AGNs (see upper panel). The numbers in the top (bottom) left of each quadrant give the number of objects in the BL and AGN 2 samples above (below) the dashed lines; for the objects above the dashed lines also the percentages have been reported.

(A color version of this figure is available in the online journal.)

et al. 2002; Mignoli et al. 2004; Brusa et al. 2005; Severgnini et al. 2005; Georgantopoulos et al. 2006).

The upper panels in Figure 6 show the $\mathrm{X} / \mathrm{O}$ value as a function of the 2-10 keV X-ray luminosity, separately for the XMMCOSMOS sources spectroscopically identified as BL AGNs (312 objects, left) and NL AGNs (244 objects, right), detected in the $2-10 \mathrm{keV}$ flux-limited sample (925 sources). The numbers in the top (bottom) left of each panel give the number of objects in the different samples above (below) the dashed lines which correspond to the thresholds of $\mathrm{X} / \mathrm{O}>10$. For the objects above the dashed lines also the percentages to the entire subsamples have been reported.

A strong correlation between the $\mathrm{X} / \mathrm{O}$ ratio and the observed $\mathrm{X}$-ray luminosity for AGN $2 \mathrm{~s}$ has been discovered by the first massive identifications campaigns in Chandra and XMMNewton surveys (e.g., F03; Eckart et al. 2006), and it is related to the fact that the X-ray luminosity is less affected by absorption than the optical luminosity. The same correlation is also present in the XMM-COSMOS sample (upper right panel of Figure 6). The dashed line is the original F03 relation, the solid line is the relation which describes our data: the slope is the same, while the zero point is different. This is mostly due to (1) the optical bands used in the $\mathrm{X} / \mathrm{O}$ parameter, $R$ band, having slightly different filter curves, and (2) most important, the average magnitude of the spectroscopically identified sample being fainter for the
XMM-COSMOS sample with respect to the sample used in F03. On the contrary, objects in the AGN 1 sample (upper left quadrant) do not present such a trend.

The lower panels of Figure 6 show the $\mathrm{X} / \mathrm{O}$ versus the 2-10 keV X-ray luminosity for the sources with photometric redshifts, separately for the objects with a best-fit SED template of unobscured quasars (model numbers SED from 19 to 30 in Salvato et al. 2009; left panel; see also Lusso et al. 2010) and for sources best fit with a galaxy-dominated template (model numbers SED $<19$ in Salvato et al. 2009; right panel). The sources in the lower right panel of Figure 6 show a correlation of the $\mathrm{X} / \mathrm{O}$ versus the $\mathrm{X}$-ray luminosity similar to that present for the AGN 2 sample, albeit with a somewhat larger scatter and higher normalization. The fact that sources at fainter optical magnitudes (as it is the case for the sources in the photo$z$ sample) have a higher normalization of the X/O versus $L_{X}$ relation is obviously expected and was already pointed out by Barger et al. (2005). The large and deep COSMOS spectroscopic database allows us to test this variation using a suitably large ( $\sim 70)$ sample of faint $(R>23)$ sources. When the sources with spectroscopic redshifts fainter than $R=23$ are considered (plotted for comparison with red symbols in the lower right panel of Figure 6), they show the same higher normalization of the $\mathrm{X} / \mathrm{O}$ versus $L_{X}$ relation. The observed correlation between $\mathrm{X} / \mathrm{O}$ and $\mathrm{X}$-ray luminosity is due to the fact that, while the nuclear AGN X-ray luminosity can span several decades, the host-galaxy $R$-band luminosity (which dominates the optical emission in obscured sources) has a much smaller scatter, less than one decade (see also Treister et al. 2005). At fainter magnitudes, lower-luminosity host galaxies are starting to be detected, moving the points toward higher $\mathrm{X} / \mathrm{O}$ values. In addition to these selection effects, it is important to note that moving the SED of a moderately obscured or Compton Thick AGN (see, e.g., Franceschini et al. 2000) to progressively higher redshifts the $K$-corrections in the optical and X-ray band work in opposite directions. The shape of the hard X-ray spectrum is responsible for a strong $K$-correction which "boosts" the $\mathrm{X}$-ray flux and favors the detection of high-redshift sources. Conversely, the weak rest-frame optical-UV emission is shifted in the $R$ band explaining the extremely faint optical magnitudes. As a consequence, the optical to X-ray flux ratio changes in a nonlinear way (see also discussion in Comastri et al. 2003) and this can explain the higher normalization observed for the fainter sources.

Figure 7 shows the $R-K$ color (Vega system) as a function of the 2-10 keV X-ray luminosity, separately for the XMMCOSMOS sources spectroscopically identified as BL AGNs and NL AGNs detected in the $2-10 \mathrm{keV}$ flux-limited sample. The numbers in the top (bottom) left of each panel give the number of objects in the different samples above (below) the dashed lines which correspond to the thresholds of $R-K>5$. The fraction of AGN 2 with high-X/O and/or red $R-K$ colors is considerably higher than the corresponding fraction for BL AGNs (e.g., about $7.5 \%$ of AGN 2 s have $R-K>5$ while only $\sim 0.9 \%$ of the BL AGNs have the same red color). When the dependence of the $R-K$ color on the X-ray luminosity for the NL AGN sample is considered, there is evidence for a trend where high-luminosity sources have redder colors than low-luminosity sources (right panel of Figure 7$)$. The median $R-K$ color for the highluminosity sample $\left(L_{X}>10^{43.5} \mathrm{erg} \mathrm{s}^{-1}\right)$ is $\langle R-K\rangle=4.17$ (0.53 dispersion), while it is $\langle R-K\rangle=3.47$ (0.62 dispersion) for the low-luminosity sample $\left(L_{X}<10^{43.5} \mathrm{erg} \mathrm{s}^{-1}\right)$. This correlation is mostly driven by redshift effects, the median 


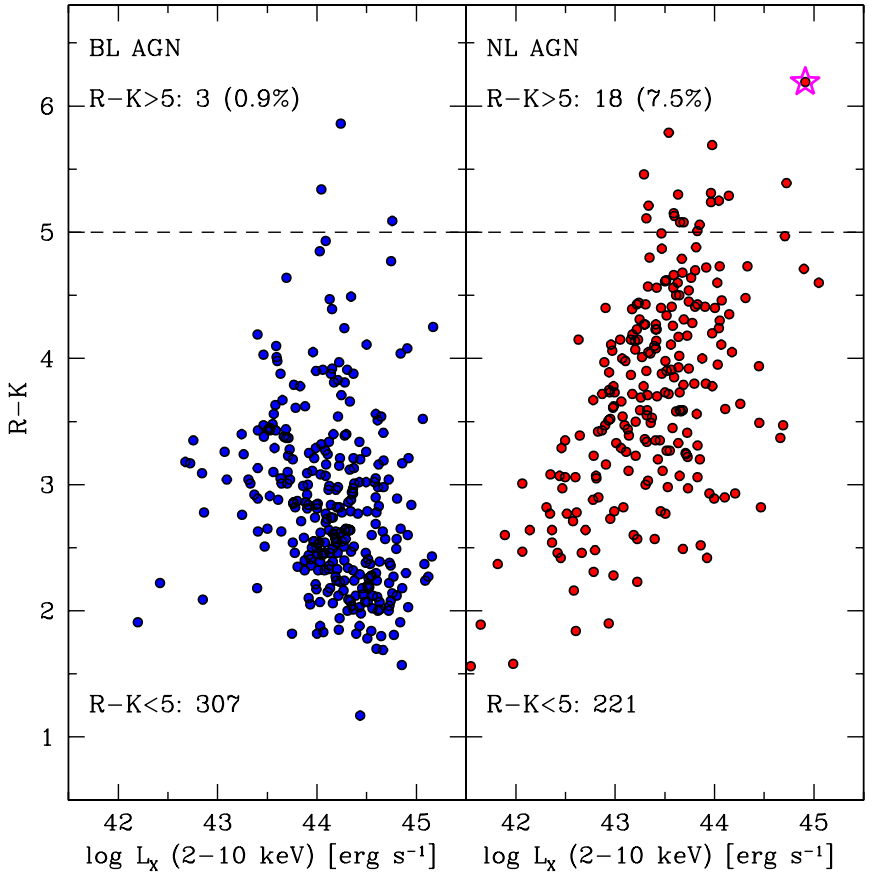

Figure 7. Left panel: $R-K$ vs. the rest-frame hard X-ray luminosity for the sources classified as BL AGNs (left, blue circles) and for the sources classified as AGN 2s (right, red circles). The magenta star marks the position in this diagram of XID 2028 (see Section 8). The numbers in the top (bottom) left of each panel give the number of objects in the BL and AGN 2 samples above (below) the dashed lines; for the objects above the dashed lines also the percentages have been reported.

(A color version of this figure is available in the online journal.)

redshifts of the two subsamples being $z=0.93$ and $z=$ 0.57 , respectively. On the other hand, lower-luminosity (lowerredshift) BL AGNs are slightly redder, as it is clear from the left panel of Figure 7. In particular, the median $R-K$ color for the high-luminosity sample $\left(L_{X}>10^{44} \mathrm{erg} \mathrm{s}^{-1}\right)$ is $\langle R-K\rangle=2.58$ (0.68 dispersion; similar to the average value of $R-K$ colors obtained from optically selected, bright quasars; Barkhouse \& Hall 2001), while it is $\langle R-K\rangle=3.04$ (0.55 dispersion) for the low-luminosity sample $\left(L_{X}<10^{44} \mathrm{erg} \mathrm{s}^{-1}\right)$. The probability that the two distributions are drawn from the same parent population is $5.5 \times 10^{-7}$ according to a Kolmogorov-Smirnov (K-S) test. Given that the main difference of the two samples is the average redshift (the low-luminosity sample being at considerably lower redshift, $z<1$, than the high-luminosity sample, $z \sim 2$ ), the most likely explanation of the observed diversity is that, at low redshift, the host galaxy dominates the rest-frame optical emission (as sampled by the $R$ and $K$ bands). These objects also present on average an extended morphology (as derived from ACS analysis; see Gabor et al. 2009) and are similar to the "extended BL AGNs" presented in B07.

The left panel of Figure 8 shows the two obscured AGN indicators discussed above plotted one against the other, e.g., the $\mathrm{X} / \mathrm{O}$ ratio versus the $R-K$ color (see also Brusa et al. 2005). In addition to the spectroscopically identified population (plotted as colored symbols), we also report as crosses the sources without spectroscopic redshifts for the whole XMMCOSMOS catalog. In the region with $R-K>5$ and $\mathrm{X} / \mathrm{O}>$ 10 , we have 105 sources, 16 with spectroscopic redshifts and 13 of them classified as AGN 2. The high rest-frame, $2-10 \mathrm{keV}$ $\mathrm{X}$-ray luminosities $\left(\log L_{X} \gtrsim 43.5 \mathrm{erg} \mathrm{s}^{-1}\right)$, further classifies these sources as quasars. For the remaining 89 sources, only photometric redshifts are available.
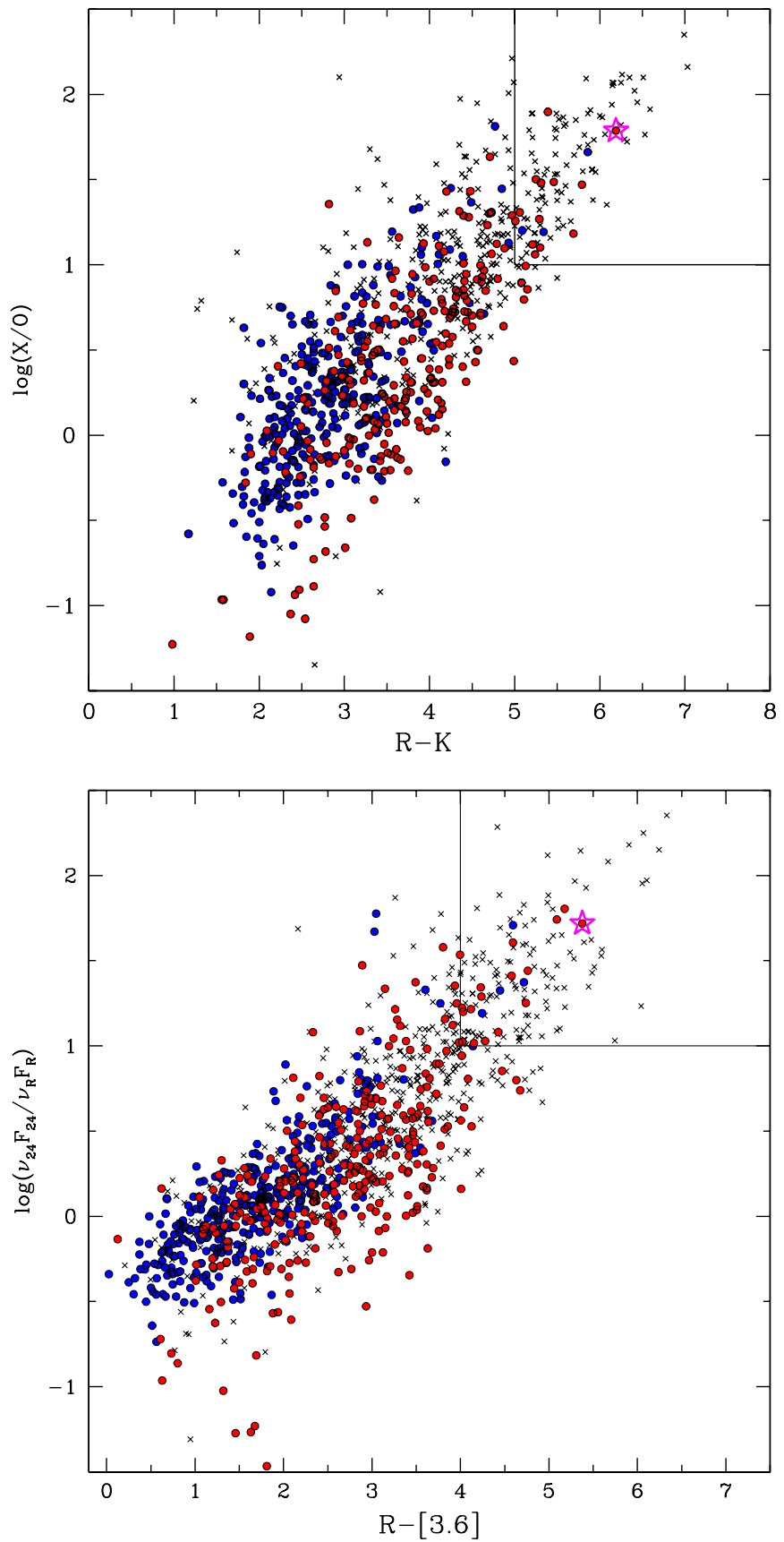

Figure 8. Left panel: the $\mathrm{X}$-ray-to-optical flux ratio $(\mathrm{X} / \mathrm{O})$ plotted against the $R-K$ color (Vega magnitude, in order to be immediately comparable with other $R-K$ selection criteria used in the literature) for the BL AGN sample (blue circles); the AGN 2 sample (red circles); and the sources with only photometric redshifts (crosses). The solid lines mark the selection for high- $z$ obscured AGN candidate used in this work $(R-K \geqslant 5$ and $\mathrm{X} / \mathrm{O}>10)$. Right panel: the ratio of the $24 \mu \mathrm{m}$ flux over the $R$-band flux vs. the $R-3.6 \mu \mathrm{m}$ color (AB magnitudes). The solid lines mark the selection criteria for high- $z$ obscured AGN candidates used in this work $\left(R-3.6 \geqslant 4\right.$ and $\left.v_{24} F_{24} / v_{R} F_{R}>10\right)$. In both panels, the magenta star marks the position of XID 2028 (see Section 8 for details).

(A color version of this figure is available in the online journal.)

\subsubsection{Mid-infrared to Optical Flux Ratio}

Recently, the combination of high MIPS $24 \mu \mathrm{m}$ to optical flux ratio and red near-infrared to optical colors has been proven to be very efficient in selecting high-redshift star-forming galaxies (Yan et al. 2005; Houck et al. 2005) and heavily absorbed, possibly Compton Thick AGNs (see Fiore et al. 2008, 2009; Dey et al. 2008; Daddi et al. 2007; Sacchi et al. 2009). Given the 


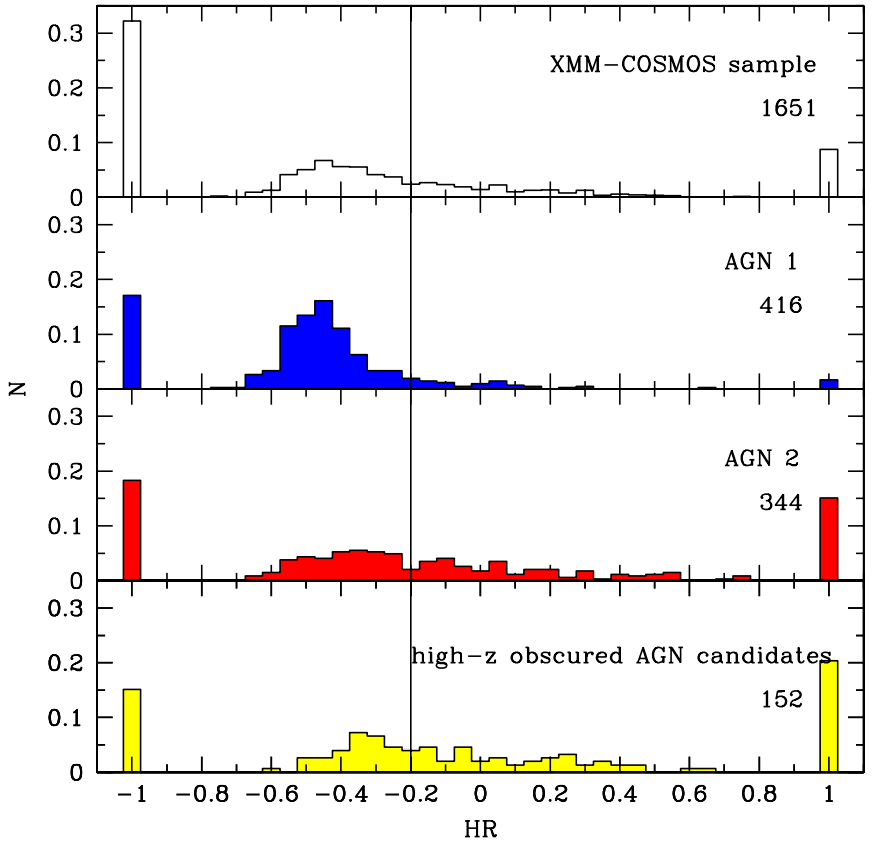

Figure 9. HR distribution normalized to the total number of sources in each sample for (a) the whole XMM-COSMOS flux-limited sample (1640 objects), (b) the AGN 1 sample (414 objects), (c) the AGN 2 sample (337 objects), and (d) the high- $z$ obscured AGN candidate as isolated from the previous figures (152 objects). The line at HR $=-0.2$ marks the threshold used in this paper to divide obscured and unobscured AGNs on a purely X-ray based classification. Sources detected only in the soft $(0.5-2 \mathrm{keV})$ or hard $(2-10 \mathrm{keV})$ band are plotted at values of $\mathrm{HR}=-1$ and $\mathrm{HR}=1$, respectively.

(A color version of this figure is available in the online journal.)

high X-ray column density, most of these sources may remain undetected even in the deepest X-ray exposures and their AGN nature can be unveiled only through stacking analysis (Daddi et al. 2007; Fiore et al. 2008, 2009; Lanzuisi et al. 2009). However, the X-ray detected population may constitute the most luminous and slightly less absorbed tail of this heavily absorbed AGN population (see also discussion in Fiore et al. 2008).

The right panel of Figure 8 shows the $v_{24} F_{24} / v_{R} F_{R}$ value versus the $R-3.6$ color for all the sources with MIPS detection in the XMM-COSMOS counterpart sample (the symbols have the same meaning as before). There are 137 sources with $v_{24} F_{24} / \nu_{R} F_{R}>10$ and $R-3.6 \geqslant 4$ (a similar criterion has been introduced by Yan et al. 2007); 64 of them are in common with the sample isolated on the basis of their combined high-X/O and $R-K$ colors (Section 7.1.1). Among the remaining 73 objects, 10 sources have spectroscopic redshifts and classifications available: 2 of them are classified as AGN 1s and the remaining 8 are classified as AGN 2s from our combined optical and $\mathrm{X}$-ray classification. For 63 sources no spectroscopic redshift information is available.

In the following, we will refer to the sample of the 152 sources $(89+63)$ without spectroscopic redshifts selected on the basis of their high-X/O and/or $\nu_{24} F_{24} / \nu_{R} F_{R}$ ratio, and red optical to near-infrared colors as the "high- $z$ obscured AGN candidates." When the spectroscopically identified population only is considered, these criteria appear to be robust in selecting obscured AGNs: only about $20 \%(5 / 25)$ of the sources satisfying these selection criteria are classified as AGN 1s, while in the XMMCOSMOS flux-limited sample the fraction of AGN 1s over the total AGN 1s+AGN 2s makes up $\sim 55 \%$. Photometric redshifts are available for $150 / 152$ sources. The redshift distribution for these high- $z$ obscured AGN candidates is plotted in the bottom right of right panel of Figure 3 (yellow histogram). This distribution does not change if we consider only the 64 sources in common between the two selections presented in 7.1.1 and 7.1.2. The vast majority of these sources lie at $z=1-3$.

\subsection{Hardness Ratio and X-ray Obscuration}

We explore in the following the X-ray properties of the high- $z$ candidate obscured AGNs and compare them with those of the entire XMM-COSMOS sample. When the number of counts in a source is inadequate to perform a spectral fit, a widely used tool to study the spectral properties of an X-ray source is the HR (see the description of Table 2 in Section 4 for the definition; e.g., Della Ceca et al. 2004; Mainieri et al. 2007). Mainieri et al. (2007) showed that the HR and the column density of the sources derived from spectral analysis are quite well correlated, and that $90 \%$ of the sources with column densities larger than $10^{22} \mathrm{~cm}^{-2}$ have $\mathrm{HR}>-0.3$. The results in Mainieri et al. (2007) have been obtained for the subsample of the XMMCOSMOS counterparts described in B07; given that the redshift distribution does not significantly differ from the one of the present sample (see Figure 5 in B07 and Figure 3 in this paper), we can rely on the $\mathrm{HR}-N_{\mathrm{H}}$ calibration as described in Mainieri et al. (2007). On the other hand, however, only about $50 \%$ of the sources with $\mathrm{HR}>-0.3$ have $N_{\mathrm{H}}>10^{22} \mathrm{~cm}^{-2}$. In order to create a cleaner sample of obscured AGNs from an HR selection, and to compare with previous results (e.g., Hasinger 2008), we use in the following a more conservative limit of $\mathrm{HR}=-0.2$ to distinguish between obscured and unobscured objects. Above this threshold, $\sim 80 \%$ of the sources have $N_{\mathrm{H}}>10^{22} \mathrm{~cm}^{-2}$.

Figure 9 shows the HR distribution, normalized to the total number of sources, for the entire XMM-COSMOS counterparts in the flux-limited sample (1640), the AGN 1 sample (416), the AGN 2 sample (344), and the high- $z$ obscured AGN candidates, as isolated in Section 7.1. As it is clear from the normalized distributions, the relative fraction of hard sources increases from top to bottom: in particular, the fraction of sources with HR $>-0.2$ in the AGN 1 sample is only $\sim 10 \%$, while it rises to $45 \%$ in the AGN 2 sample and up to $53 \%$ in the high- $z$ obscured AGN candidates sample (80/152). The median values of the HR in the three different classes are $\mathrm{HR}=-0.49$ for the BL AGN, $\mathrm{HR}=-0.29$ for the NL AGN, and $\mathrm{HR}=-0.17$

The average HR values of the two obscured AGN samples (NL AGN and high- $z$ obscured AGN candidates) are not directly comparable, given that the average redshifts of the two samples are different (with median values of $\langle z\rangle=0.73$ and $\langle z\rangle=1.72$, respectively) and the $N_{\mathrm{H}}-\mathrm{HR}$ relation is redshift dependent. In addition, it is worth noting that, if the NL AGN sample is significantly contaminated by misclassified low-luminosity BL AGNs for which the broad-line emission is diluted in the hostgalaxy light (see Section 7.1 and Section 7.3), the average HR value may be contaminated too, resulting in an average softer value than the intrinsic one. However, it is also worth pointing out that the redshift effect works against hard HR at high- $z$ and, therefore, the fact that high- $z$ obscured AGN candidates are harder than NL AGNs confirms the efficiency of the proposed selection in picking up the most obscured sources.

\subsection{IRAC Color-Color Diagrams and Color Indices}

Since the advent of the Spitzer satellite, several color-color diagrams based on the combination of flux ratios from the 


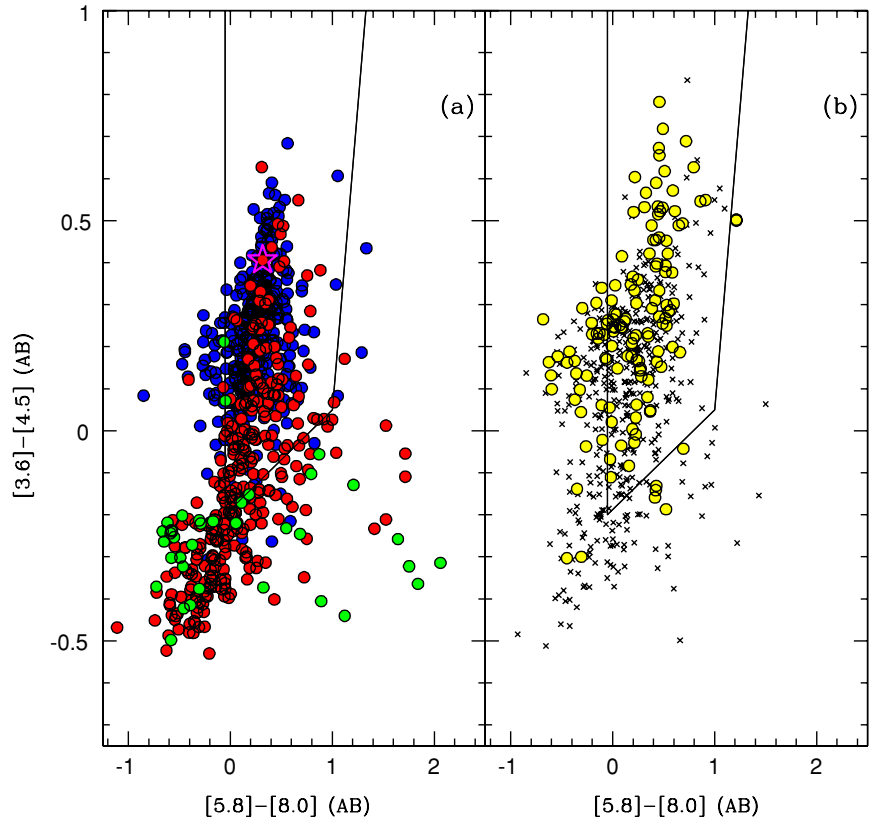

Figure 10. (a) Stern color-color diagram for the spectroscopically identified sample. Symbols and colors as in previous figures. (b) Stern color-color diagram for the sources with only photometric redshifts ( $\sim 600$ sources, crosses) and for the high- $z$ obscured AGN candidates ( $\sim 150$ sources, yellow points).

(A color version of this figure is available in the online journal.)

four different IRAC channels have been widely used to classify infrared sources and isolate obscured AGNs missed in optical and near-infrared surveys (Lacy et al. 2004; Stern et al. 2005; Hatzminiaoglou et al. 2005; Barmby et al. 2006).

Figure 10 shows the IRAC color-color diagram (as initially proposed by Stern et al. 2005) for the sources in the XMMCOSMOS counterpart sample. For this analysis only sources with IRAC detection in all the four bands and with good determination of the photometry (i.e., not in masked region, with error $<25 \%$ of the IRAC flux) are considered, for a total of 1326 objects. Panel (a) shows the distribution in this diagram of the spectroscopically identified population, for the three different categories described in Section 5.1: 380 AGN 1s (blue circles), 296 AGN 2s (red circles), and 37 normal galaxies (green circles). The locus at [5.8]-[8.0] $>1$ and [3.6]-[4.5] $<0$ mainly selects X-ray sources which are expected to be lowredshift normal and star-forming galaxies (see also discussion in Feruglio et al. 2008). The dashed lines isolate the wedge for the selection of luminous quasars, originally proposed in Stern et al. (2005). While $90 \%$ of the BL AGNs are successfully recovered from the IR selection, and reassuringly almost all $(95 \%)$ of the normal galaxies detected in the X-rays lie indeed outside the wedges, only $48 \%$ of the X-ray selected non-broadline AGNs lie within the Stern's wedge. Similar results can be obtained using IRAC-based color-selection wedges different from the specific one proposed by Stern et al. (2005), e.g., those proposed by Lacy et al. (2004) or Hatzminiaoglou et al. (2005). This analysis confirms that purely IRAC-based criteria are not optimally designed to select AGNs in which the host galaxy dominates the near-infrared energy (e.g., lowerluminosity AGNs), and/or AGNs obscured in the IRAC bands, and therefore may miss a significant fraction of the lowluminosity, obscured AGN population. Most of the non-broadline AGNs outside the wedge are, indeed, moderate luminosity objects at intermediate redshifts $(z<1$; see also Cardamone et al. 2008; Georgantopoulos et al. 2008; Brusa et al. 2009b), in which the host-galaxy light dominates the IR emission.

Panel (b) shows the same diagram for the population with photometric redshifts only (572 sources). Superimposed as yellow circles are the high- $z$ obscured AGN candidates isolated through the exploitation of the multiwavelength properties described in the previous sections. The vast majority $(80 \%)$ of the high- $z$ obscured AGN candidates would have been isolated also through the IRAC selection, confirming that these sources are luminous AGNs, with the NIR/MIR emission dominated by the central engine. However, at the limiting fluxes of the IRAC COSMOS survey the contamination from high-redshift $(z>1)$ normal and star-forming galaxies in the Stern et al. (2005) wedge starts to become important (see discussion in Sajina et al. 2005) and the selection criterion would become efficient only when combined with an additional criterion (in this case, the X-ray emission).

It is interesting to note that a nonnegligible fraction $(\sim 18 \%)$ of the high- $z$ obscured AGN candidates lie at [3.6]-[4.5] $>0$ $(\sim 0.25)$ and [5.8]-[8.0] $<0$, and would be missed by IRAC selections based on AGN SED templates, like the one proposed by Stern et al. (2005). As a comparison, the fraction of objects satisfying the same selection for the spectroscopically identified population is only $\sim 5 \%$. This locus is where both star-forming and normal galaxies colors at $z \sim 1-3$ are expected (see Barmby et al. 2006). Therefore, in our X-ray selected AGN sample, also this locus is populated by objects where the host galaxy dominates and/or the AGN is obscured up to $8 \mu \mathrm{m}$ $(\sim 2.5 \mu \mathrm{m}$ rest frame). Given the high inferred X-ray luminosity $\left(L_{X} \gtrsim 10^{43.5} \mathrm{erg} \mathrm{s}^{-1}\right)$, this also means that these objects should have a luminous (and massive) host, in agreement with the results of Mignoli et al. (2004), Severgnini et al. (2005), and others.

The IRAC broadband color ([3.6]-[8.0]) can be used to disentangle sources with an infrared-rising (red) SED, e.g., AGNs, power-law dominated sources, and sources with an inverted (blue) infrared SED, with more flux at shorter wavelengths than at longer wavelengths, e.g., normal galaxies at low- $z$ (see also discussion in Barmby et al. 2006). Sources with a flat luminosity per given wavelength range ( $\lambda f_{\lambda}=$ constant) have [3.6]-[8.0] $\sim 0.86$. Figure 11 shows the distribution of the [3.6]-[8.0] color for the entire XMM-COSMOS population (black histogram), the AGN 1 sample (blue shaded histogram), the AGN 2 sample (red shaded histogram), and the high- $z$ obscured AGN candidates sample (yellow shaded histogram). AGN 1s populate the red power-law part of the distribution, as expected, with a median value of $\langle[3.6]-[8.0]\rangle=1.46$ (0.34 dispersion). On the contrary, the XMM-COSMOS sample of spectroscopically identified AGN 2s (limited mainly at $z<1$ and to moderate luminosity objects) preferentially show IRAC colors consistent with an inverted power law, i.e., in the host-galaxy-dominated regime (the median value of the distribution is $\langle[3.6]-[8.0]\rangle=0.65$ and a wider dispersion, 0.55$)$. The probability that the two distributions are drawn from the same parent population is $<10^{-7}$ according to a K-S test. The high- $z$ obscured AGN candidates set in between the two spectroscopically selected samples, around the median value $\langle[3.6]-[8.0]\rangle=1.28$ (0.55 dispersion), with only $\sim 25 \%$ of the sources having [3.6]-[8.0] $<0.865$. This is a further confirmation that the devised strategy is efficient in selecting luminous AGNs with red power-law (i.e., AGN dominated) SED but elusive in the optical bands. 


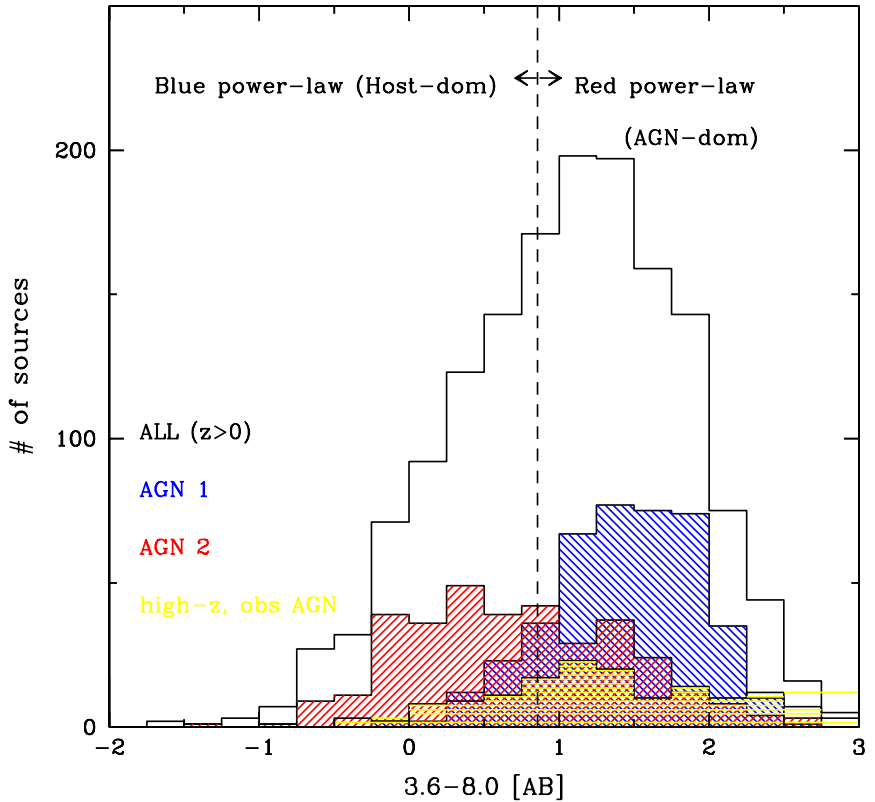

Figure 11. Distribution of the [3.6]-[8.0] color for the XMM-COSMOS counterparts, shown separately for the entire extragalactic sample (black), the AGN 1 sample (blue), the AGN 2 sample (red), and the high- $z$ obscured AGN candidate sample (yellow). The dashed line at [3.6]-[8.0] $=0.856$ marks the division between "blue" and "red" power-law sources.

(A color version of this figure is available in the online journal.)

\section{LUMINOSITY DEPENDENCE OF THE OBSCURED AGN FRACTION}

The large sample of X-ray sources in XMM-COSMOS can be used to explore the luminosity dependence of the obscured AGN fraction. In Figure 12, we plot the fraction of obscured AGNs (defined as the fraction of objects classified as NL AGN over the total number of AGN sources) in different luminosity bins for the spectroscopically identified hard X-ray selected XMM-COSMOS sample (open squares). This fraction is a strong function of the X-ray luminosity, being almost $90 \%$ at $L_{X} \sim 10^{42} \mathrm{erg} \mathrm{s}^{-1}$ and $<10 \%$ at $L_{X} \sim 10^{45} \mathrm{erg} \mathrm{s}^{-1}$.

It is important to note, however, that, even if the spectroscopic sample is representative of $\sim 60 \%$ of the XMM-COSMOS source population, high-redshift (i.e., high-luminosity) obscured AGNs are optically faint (typically $I \sim 23-24$ or fainter) and have not been targeted yet with dedicated spectroscopic campaigns. The resulting fraction at high luminosity is therefore expected to be biased, in particular against the obscured objects (see discussion in previous subsection). In order to extend the study of the AGN fraction as a function of the luminosity to the full XMM-COSMOS sample, and reduce the bias against high-luminosity obscured AGN, we calculated the fraction of obscured AGN fully exploiting the information contained in the multiwavelength catalog. For sources with spectroscopic redshifts, we used the optical/X-ray classification as defined in Section 5.1; for sources without spectroscopic redshifts, we used the classification from Salvato et al. (2009) based on the best-fitting SED procedure (see also Section 7.1), which turned out to be well matched with the optical classification and $\mathrm{X}$-ray HRs from X-ray color diagrams (see discussion in Salvato et al. 2009 and C09): sources with best-fit SED templates of unobscured quasars (model numbers SED from 19 to 30 in Salvato et al. 2009; see also Lusso et al. 2010) are classified as Type 1 (unobscured) AGNs, while sources best-fit with a galaxy-

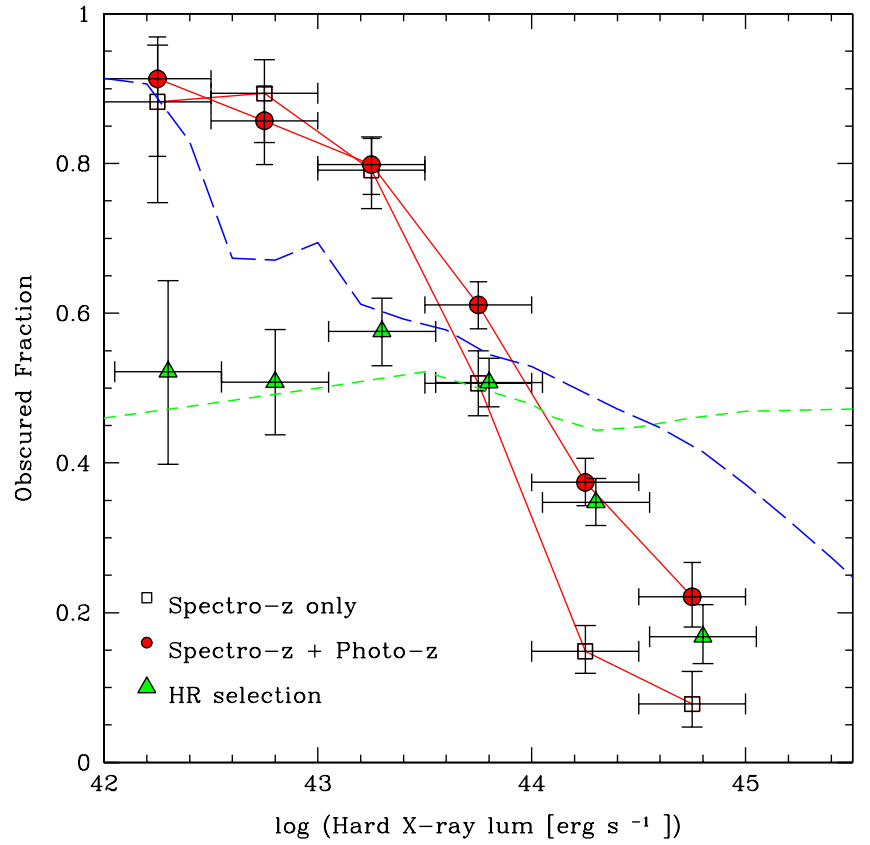

Figure 12. Ratio of obscured to total AGNs as a function of hard X-ray luminosity. The open squares with error bars show the obscured fraction computed from the sources in the $2-10 \mathrm{keV}$ sample with spectroscopic information ( $\sim 550$ objects). The red filled circles represent the fraction obtained for the full 2-10 keV sample, where the classification in obscured and unobscured sources is made on the combination of spectroscopic information and the best-fit SED template from sources without spectroscopic redshifts ( $\sim 900$ objects). The green triangles mark the fraction computed when a purely X-ray selection (based on the HR) is adopted. The green short-dashed and blue long-dashed lines represent the predictions on the fraction of AGN with $N_{\mathrm{H}}>10^{22} \mathrm{~cm}^{-2}$ from the XRB synthesis models by Gilli et al. (2007) and Treister \& Urry (2006), respectively. Errors are $1 \sigma$, calculated following Gehrels (1986).

(A color version of this figure is available in the online journal.)

dominated template (model numbers SED $<19$ in Salvato et al. 2009) are classified as Type 2 (obscured) AGNs. We then computed a new estimate of the fraction of obscured AGN as a function of X-ray luminosity (red circles in Figure 12). Not surprisingly, the fraction of obscured AGN derived in this way at $L_{X}>10^{44} \mathrm{erg} \mathrm{s}^{-1}$ is considerably higher (a factor of $\sim 2-3$ ) than that computed for the spectroscopically identified sample.

Finally, we computed the obscured fraction derived by dividing sources in obscured and unobscured on the basis of the $\mathrm{X}$-ray HR, which can be used as a crude indicator of the X-ray (nuclear) obscuration, although suffering from large uncertainties (see, e.g., Tozzi et al. 2006 and Section 7.2). We chose as threshold for classifying an object as an obscured AGN the value $\mathrm{HR}=-0.2$ (corresponding to $N_{\mathrm{H}} \sim 10^{22} \mathrm{~cm}^{-2}$ for a $\Gamma=1.8$ spectrum), as discussed in Section 7.2. The results are plotted as green triangles in Figure 12. All of the obscured AGN fraction estimates refer to observed quantities and have not been corrected for selection effects.

In Figure 12, we also compare our different estimates of the obscured AGN fraction as a function of the luminosity derived as outlined above with those predicted by the XRB synthesis models of Gilli et al. (2007; from an X-ray based classification, long-dashed green curve) and Treister \& Urry (2006; from a combined optical and X-ray classification, shortdashed blue curve), for the $N_{\mathrm{H}}>22 \mathrm{~cm}^{-2}$ population, folded with the survey sky coverage. In the luminosity range $\log L_{X}=$ 43.5-44.5 $\mathrm{erg} \mathrm{s}^{-1}$, i.e., around $L_{*}$ luminosity for the XMM- 
COSMOS sample sources, the two predictions are broadly consistent with each other and with the observed estimates derived from the HR and from the combined (spectroscopic plus SED) optical classification. At low luminosities $\left(\log L_{X}<\right.$ $43 \mathrm{erg} \mathrm{s}^{-1}$ ), optically based classifications seem to yield higher obscured AGN fractions. Similarly, XRB models predictions largely differ: the Gilli et al. (2007) curve well trace the X-ray based estimate of the obscured AGN fraction, while the Treister \& Urry (2006) model predictions are closer to the estimate from optical classification. ${ }^{37}$ This reflects the paucity of BL AGNs in the XMM-COSMOS sample at low- $L\left(\log L_{X}<43 \mathrm{erg} \mathrm{s}^{-1}\right.$, see Figures 6 and 7) and might be due, at least in part, to the effect of the host-galaxy contribution in the observed spectral range. Indeed, broad line signatures from low-luminosity AGNs may get diluted in the host-galaxy emission (see discussion in Caccianiga et al. 2007; Civano et al. 2007), and this effect is likely to be important at low Eddington ratios and at low luminosities (Hopkins et al., 2009). At higher X-ray luminosities $\left(\log L_{X}>44.5 \mathrm{erg} \mathrm{s}^{-1}\right)$ both models overpredict the observed obscured fraction by a factor of $\sim 2-3$.

The luminosity dependence of the obscured AGN fraction can also explain why low-luminosity AGNs lie preferentially outside the IRAC selection wedges populated by spectroscopically selected unobscured quasars: the lower the luminosity, the higher is the percentage of obscured sources and therefore on average the higher is the contribution from host galaxy on the observed SED and colors, which eventually drives the galaxies outside the selection wedges (see, e.g., Sajina et al. 2005; Barmby et al. 2006; Georgantopoulos et al. 2008).

\section{A PROTOTYPICAL OBSCURED QSO AT $z=1.59$}

An enhancement in the bolometric luminosity and column density, coupled with a low $B$-band luminosity (and therefore red optical to near-infrared colors, and high-X/O and MIPS/O flux ratios), as well as strong outflows from AGNs and/or stellar winds are predicted for objects that are experiencing a transition from being starburst dominated to AGN dominated by most recent models of AGN galaxy coevolution (Menci et al. 2008; Hopkins et al. 2008; Hickox et al. 2009; see also Narayanan et al. 2009).

Almost by definition, most of the high- $z$ obscured AGN candidates isolated Section 7 are expected to be undergoing this peculiar phase of their evolution. The most extreme object in the spectroscopically identified sample which satisfies the selection criteria described in Section 7 is XID $2028(z=1.592)$, marked with a magenta star in the previous diagnostics (Figures 6-8). This is the object with the reddest optical color $(R-K=6.46)$ and the second highest X-ray-to-optical $(\log X / O=1.79)$ flux ratio in the spectroscopically identified sample. The high $\mathrm{X}$ ray luminosity $\left.\left(L_{X}\right) \sim 10^{45} \mathrm{erg} \mathrm{s}^{-1}, 2-10 \mathrm{keV}\right)$ and the high column density derived from the spectral analysis $\left(\log N_{\mathrm{H}}=\right.$ $22.0 \pm 0.1 \mathrm{~cm}^{-2}$; V. Mainieri et al. 2010, in preparation ${ }^{38}$ ) classify this object as an obscured Type 2 Quasar. The high

\footnotetext{
37 At variance with Treister et al. 2009a, for the open squares we use only a spectroscopic classification. However, we note that the hybrid classification in Treister et al. (2009a) is used only for sources at $z<0.5$, which translates to $L_{X}<10^{42.5} \mathrm{erg} \mathrm{s}^{-1}$ at the limiting flux of XMM-COSMOS sources, and $L_{X}<10^{43} \mathrm{erg} \mathrm{s}^{-1}$ at the limiting flux of the ECDFS sample, i.e., the first two bins in Figure 12. The results at higher X-ray luminosities $\left(L_{X}>10^{43} \mathrm{erg} \mathrm{s}^{-1}\right.$ ) can therefore be considered comparable.

38 In Mainieri et al. (2007), this object has been assigned a lower redshift, $z=0.784$, on the basis of a lower-quality optical spectrum available at that time. The higher redshift translates in a higher column density than the one reported in Mainieri et al. (2007), $\log N_{\mathrm{H}}=21.83$.
}
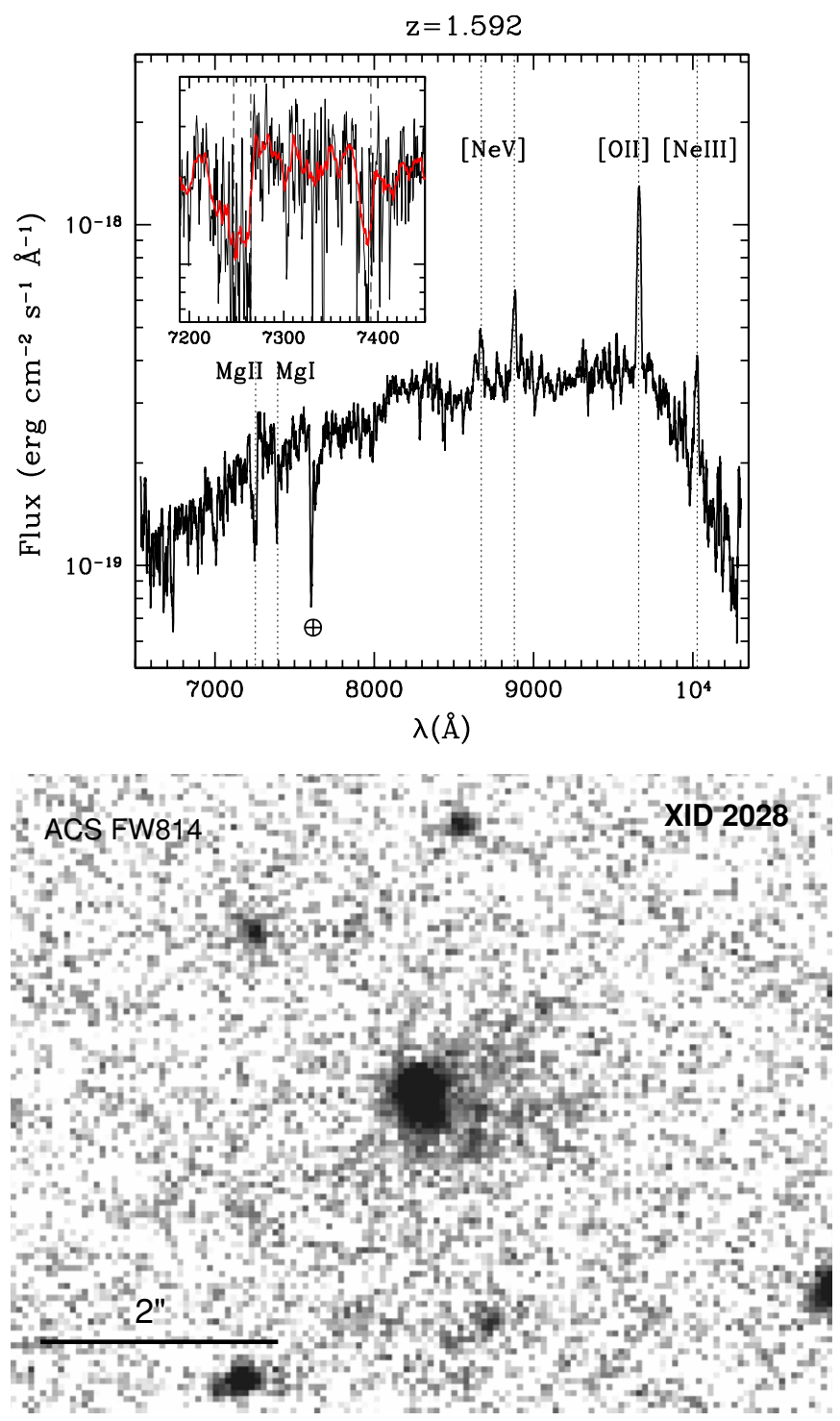

Figure 13. Left panel: observed-frame Keck spectrum of XID 2028, with superimposed the various emission and absorption lines (dotted vertical lines, as labeled); the inset shows the zoom on the $\mathrm{Mg}$ II and $\mathrm{Mg}$ I lines. The black curve shows the unbinned data, while the red curve shows the smoothed spectrum (boxcar of 15). The vertical dashed lines show the expected positions of the absorption lines at the redshift derived from the $\mathrm{O}[\mathrm{II}]$ emission line. Right panel: HST/ACS image centered around XID 2028.

(A color version of this figure is available in the online journal.)

mid-infrared to optical flux ratio $\left(v_{24} F_{24} / \nu_{R} F_{R}>50\right)$ further classifies XID 2028 as a dust obscured galaxy (DOG; see Dey et al. 2008; Fiore et al. 2008).

The optical spectrum for this source was obtained with DEIMOS (in MOS mode) at the Keck-II telescope (Faber et al. 2003) on 2008 January 8 under mostly clear conditions with seeing of $\sim 1^{\prime \prime}$. The data were collected with the $8301 \mathrm{~mm}^{-1}$ grating tilted to $8300 \AA$ and the OG550 blocker, with a resolution of $2.5 \AA$. The spectra were dithered $\pm 2^{\prime \prime}$ along the slit to remove ghosting (internal reflections) from the $8301 \mathrm{~mm}^{-1}$ grating. Each exposure was 20 minutes for a total of $2.6 \mathrm{hr}$. The spectrum (5500-10000 $\AA$ ) is shown in the left panel of Figure 13. It presents a red, host-galaxy-dominated continuum superimposed with high-ionization narrow emission lines typical of obscured AGNs (e.g., [Ne v] $\lambda \lambda 3426,3346$ doublet), starburst indicators (e.g., [O II] $\lambda 3727$ ), and $\mathrm{Mg}$ II $\lambda 2798$ and $\mathrm{Mg}_{\mathrm{I}} \lambda 2852$ lines in 

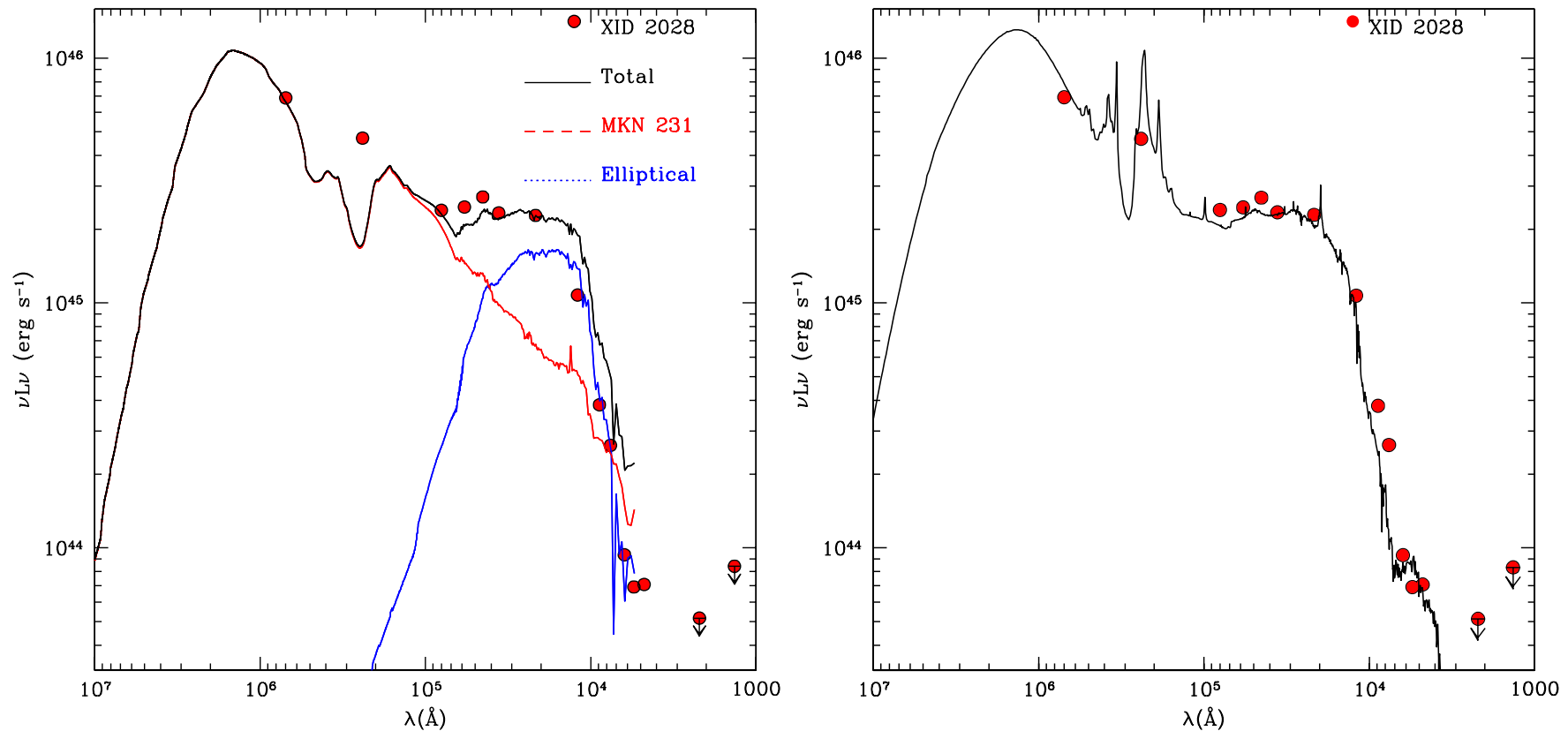

Figure 14. SED from the radio to the UV (GALEX) of XID 2028. In the left panel, the red line is the SED of Mrk 231 while the blue line is the SED of an elliptical galaxy 2 Gyr old (both SEDs are from Polletta et al. 2007). The black line is the sum of the two SEDs combined in order to provide a good representation of the SED of XID 2028. In the right panel, the black template is taken from Narayanan et al. (2009). See the text for details.

(A color version of this figure is available in the online journal.)

absorption. The redshift $z=1.592$ has been derived from the [O II] emission line and it is consistent (within $0.1 \%$ ) with that measured from the other three emission lines visible in the spectrum. The inset in the left panel of Figure 13 shows a zoom of the spectrum (black: unbinned data, red: smoothed data, with a 15 pixel boxcar) around the $\mathrm{Mg}$ absorption complex. The Mg II and $\mathrm{Mg}$ I absorption lines are slightly blueshifted with respect to the systemic velocity defined by [O II] (dashed lines in the inset) indicating the possible presence of outflows in the system (see, e.g., Weiner et al. 2009). The measured offset $(\sim 6 \AA)$ implies a velocity of $\sim 300 \mathrm{~km} \mathrm{~s}^{-1}$ when the uncertainties in the wavelength scale and in the redshift measurements $(\sim 3 \AA)$ are taken into account. Although we cannot uniquely assess which is the process which drives the outflowing material, due to the concomitant presence of high star formation and AGN activity in this system, it is interesting to note that the value observed for XID 2028 is slightly larger than what is generally observed for star formation driven outflows $\left(\sim 150 \mathrm{~km} \mathrm{~s}^{-1}\right.$; Shapley et al. 2003).

The SFR derived from the [O II] emission applying the lowredshift calibration (Kennicutt 1998) is $\sim 10^{3} M_{\odot} \mathrm{yr}^{-1}$. XID 2028 is the highest-redshift X-ray selected Type 2 Quasar detected in the $70 \mu \mathrm{m}$ survey of the COSMOS field (Kartaltepe et al. 2010). The high $70 \mu \mathrm{m}$ flux implies an infrared luminosity greater than $10^{13} L_{\odot}$, further classifying this source as a hyper luminous infrared galaxy (HyLIRG; Sanders et al. 1988), with an SFR of few $\times 10^{3} M_{\odot} \mathrm{yr}^{-1}$, in agreement, within a factor of $2-3$, with the one estimated from the optical spectrum. However, this value is likely to be an upper limit given that the [O II] emission and the $70 \mu \mathrm{m}$ emission (which at the redshift of the source corresponds to the rest-frame $\sim 25 \mu \mathrm{m}$ emission) can be severely contaminated by AGN activity (see discussions in Silverman et al. 2009a; Daddi et al. 2007).

The broadband SED (70 $\mu \mathrm{m}$ Spitzer to UV, including the Galaxy Evolution Explorer (GALEX) points retrieved from Zamojski et al. 2007) of XID 2028 is reported in Figure 14.
The left panel shows the XID 2028 SED with superimposed the template of Mkn 231, a well-known Compton Thick AGNULIRG system (red curve) and the template of an elliptical galaxy (2 Gyr old, blue curve) both taken from Polletta et al. (2007). The sum of the above templates (black) is shown in order to illustrate that the SED of XID 2028 is likely to be the result of the combination of a massive, luminous, host galaxy which dominates the optical light $\left(M_{*} \sim 5 \times 10^{11} M_{\odot}\right.$, assuming $M / L=0.22$ from Ilbert et al. 2010), with a starburst/AGN component, seen unblocked in the IR bands. The composite AGN/starburst nature of this source is also revealed in optical morphology. The right panel of Figure 13 shows the high resolution Hubble Space Telescope (HST)/ACS image cutout centered on XID 2028. From the image, a point-like nucleus is clearly visible, likely responsible for the X-ray emission, as well as a residual diffuse component (i.e., the host galaxy or a merger remnant).

The SED of XID 2028 is shown again in the right panel of Figure 14, this time superimposed with a numerical (representative) SED resulting from the models for the $z \sim 2$ DOG population published by Narayanan et al. (2009). Apart for a scaling of the overall normalization of the system ( 0.66 dex higher), this template reproduces almost perfectly the observed data points. The stellar mass used as input parameter of the numerical models ranges from $M_{*} \sim(4.7-5.3) \times 10^{11} M_{\odot}$, almost coincident with the value derived from the SED fitting. The input $M_{\mathrm{BH}}$ is also available from the simulations, and it ranges from $7 \times 10^{8}$ to $10^{9} M_{\odot}$.

The bolometric luminosity can be estimated from the X-ray emission assuming an appropriate bolometric correction (Elvis et al. 1994 or more recently Lusso et al. 2010), and it is of the order of $\sim 2 \times 10^{46} \mathrm{erg} \mathrm{s}^{-1}$, consistent with the bolometric luminosity obtained from the overall observed SED (dominated by the IR emission). Assuming that for XID 2028 the energy is released in a radiatively efficient way $\left(L / L_{\mathrm{Edd}} \sim 0.1-1\right)$, as expected for objects in the transitioning phase described 
above, it is therefore possible to derive the $\mathrm{BH}$ mass under the Eddington limited accretion scenario. This translates into a BH mass of the order of $\sim 10^{8}-10^{9} M_{\odot}$, in agreement with what can be inferred from the host-galaxy mass assuming the local $M_{\mathrm{BH}}-M_{\mathrm{Bulge}}$ relation and its evolution (Gültekin et al. 2009; Merloni et al. 2010; Jahnke et al. 2009), and within the range of the input BH mass assumed in the numerical SED simulations.

A comparison between the broadband properties of XID 2028 with those of other luminous, obscured quasars reported in the literature shows some similarities, but also remarkable differences. The SED of other X-ray selected luminous obscured quasars (e.g., Mainieri et al. 2005; Severgnini et al. 2006; Le Floc'h et al. 2007; Pozzi et al. 2007; Vignali et al. 2009) shows evidences favoring the presence of a massive starburst component and obscured accretion, with similar optical and $\mathrm{X}$-ray spectra. However, we also note that the optical spectrum of XID 2028 is quite different from that of the high- $z$, Type 2 QSO prototype CXO 202 discovered in the CDFS (Norman et al. 2002; at $z=3.7$ ). In the Norman et al. (2002) object, the optical spectrum is dominated by narrow emission line with almost no underlying continuum, while the host-galaxy contribution is clearly visible and dominant in XID 2028. However, CXO 202 is at much higher redshift than XID 2028, and it is therefore well possible that XID 2028 would show some of the same high-ionization lines and less continuum were it at higher redshift (and vice versa). Also, the different level of obscuration $\left(\sim 10^{22} \mathrm{~cm}^{-2}\right.$ for XID 2028 versus $>10^{24} \mathrm{~cm}^{-2}$ for CXO 202) may cause the differences we see in the optical spectra. In any case, the observations above highlight the power of a full multiwavelength approach to fully characterize a key phase in AGN evolution.

\section{CONCLUSIONS}

We presented the catalog of optical and infrared identifications for $\sim 1800 \mathrm{X}$-ray sources detected in the XMM-COSMOS survey. This catalog comprises the totality of the $X M M$ sources presented in $\mathrm{C} 09$, with the exception of 65 faint sources $(\sim 3 \%)$ detected in two additional fields obtained in AO6. The optical/ IR matches with the X-ray positions were driven by the Chandra subarcsec centroids, when available, or estimated through statistical methods already successfully tested in different $X M M$ and Chandra fields (see B07; Cardamone et al. 2008). For each source, a flag on the reliability of the X-ray-to-optical/infrared association is provided, classifying the counterparts as "reliable" (87.7\%), "ambiguous" (11.3\%), and "not identified" (1\%; see Table 1). For sources with two possible associations both entries are reported in the associated catalog (Table 2). The availability of Chandra data allowed us also to quantify the reliability of the likelihood ratio technique when applied to $X M M$ sources matched to deep optical and infrared images, which turned out to be very high ( $\gtrsim 98.5 \%$; see Section 3.1).

Along with the positions of the optical counterparts, we publish a wealth of multiwavelength information, from $24 \mu \mathrm{m}$ to X-ray (see Section 4 and Table 2), most notably a collection of all the available spectroscopic redshifts $(\sim 900)$ obtained through different programs ongoing in the COSMOS field (see Section 5 for details). To maximize the completeness over a well-defined large area and, at the same time, keep selection effects under control, for the multiwavelength analysis presented in this paper we further considered the subsample of 1640 identified sources detected above the limiting fluxes corresponding to a coverage of more than $1 \mathrm{deg}^{2}$ in at least one of the three X-ray energy ranges considered (see Section 3.1). The multiwavelength properties of the fainter X-ray sources are investigated in a much greater detail in the framework of the C-COSMOS survey (Elvis et al. 2009; F. Civano et al. 2010, in preparation).

The spectroscopic completeness in the soft $(0.5-2 \mathrm{keV})$ and hard (2-10 keV) subsamples is $\sim 50 \%$ and $\sim 60 \%$, respectively, remarkably high for identification campaigns of X-ray sources, to be compared with the spectroscopic completeness in the AEGIS survey ( $40 \%$ in the $2-10 \mathrm{keV}$ band; see Aird et al. $2010)$; in the X-Bootes survey ( 50\%; R. Hickox 2010, private communication; see also Murray et al. 2005; Kenter et al. 2005); and in the CDFS 2 Ms sample $(<50 \%$; Luo et al. 2008; Luo et al. 2010). By far, the highest spectroscopic completeness is for the sources detected in the ultra-hard $(5-10 \mathrm{keV})$ band, where it reaches $\sim 80 \%$ : for the first time, XMM-COSMOS provides a statistically significant sample (221) of sources detected at $>5 \mathrm{keV}$ with a very high spectroscopic breakdown at fluxes $>10^{-14} \mathrm{erg} \mathrm{cm}^{-2} \mathrm{~s}^{-1}$. As a comparison, the BeppoSAX HELLAS survey had a $50 \%$ completeness $(71 / 147)$ at higher X-ray fluxes $\left(>3 \times 10^{-14} \mathrm{erg} \mathrm{cm}^{-2} \mathrm{~s}^{-1}\right.$; Vignali 2001), while the $X M M$-HBSS has an almost complete (97\%) spectroscopic information, but it is limited to 62 sources at fluxes $>7 \times 10^{-14} \mathrm{erg} \mathrm{cm}^{-2} \mathrm{~s}^{-1}$ (Della Ceca et al. 2008).

For the sources without spectroscopic redshifts, accurate photometric redshifts (down to $\sigma(\Delta z /(1+z)$ ) $\sim 0.01$ at $I<22.5)$ are available (Salvato et al. 2009), allowing an almost $100 \%$ complete sample of X-ray sources with redshift information. This, coupled with the large number of objects, allowed us to sample with an unprecedented statistic the highluminosity tail of the X-ray luminosity function. The present data allow us to derive the AGN space density as obtained by the COSMOS survey over a relatively broad redshift range $(z \sim 1-3)$ and luminosity interval $\left(\log L_{X}=44.5-47\right)$. We compared the COSMOS results with those discussed in recent papers (Ebrero et al. 2009; Yencho et al. 2009; Aird et al. 2010) combining data from different surveys and covering in a fairly homogeneous way the luminosity-redshift plane. Our results suggest that at high luminosities $\left(\log L_{X}>44.5\right)$, the AGN number density peaks at $z \sim 2-2.5$, in good agreement with LDDE parameterization of the above-mentioned XLF.

We investigated the dependence of the X-ray-to-optical flux ratio versus the X-ray luminosity for BL AGNs and NL AGNs, and sources with photometric redshifts only. The normalization of the correlation between $\mathrm{X} / \mathrm{O}$ and $\mathrm{X}$-ray luminosity for the obscured AGNs increases going from the spectroscopically identified sample to the objects with only photometric redshifts available (i.e., toward fainter magnitudes; see Figure 6). The well-known relation between the $\mathrm{X} / \mathrm{O}$ and the $\mathrm{X}$-ray luminosity (F03; Barger et al. 2005) for obscured AGNs is mostly driven by the fact that, while the nuclear AGN X-ray luminosity can span several decades (depending on the BH mass and on the accretion rate), the host-galaxy $R$-band luminosity (which dominates the optical emission in obscured sources) has a moderate scatter, around the $M_{\mathrm{BH}}-M_{\text {Bulge }}$ relation, i.e., depending only on the $\mathrm{BH}$ mass.

We then studied the dependence of the $R-K$ color on the $\mathrm{X}$-ray luminosity and we found an opposite behavior for BL AGNs and NL AGNs in our sample. For NL AGNs, there is evidence for a trend where high-luminosity sources have redder colors than low-luminosity sources (see Figure 7). This correlation is most likely due to redshift effects, given that the $R-K$ color of normal and star-forming galaxies increases up to redshift $z \sim 2$. On the contrary, while high-luminosity 
BL AGNs show a median $R-K$ color consistent with that observed in optically selected samples (Barkhouse \& Hall 2001), lower-luminosity BL AGNs show a median $R-K$ color statistically different (redder) from the one observed for the higher-luminosity ones (see Figure 7). This difference in the $R-K$ colors for high- and low-luminosity sources is likely due to the contribution of the host galaxies in the latter sample. We conclude that X-ray surveys are effective in isolating also unobscured objects otherwise missed by optical multicolor surveys, with preselection on the basis of optical colors and point-like emission (see also similar conclusions obtained on BL AGN samples from pure spectroscopic selection, Bongiorno et al. 2007). However, the effect of host-galaxy contribution may play an important role in getting the correct classification of the nuclear source.

The large body of COSMOS multiwavelength data allowed us to devise a robust method to build a sizable sample of luminous obscured AGN candidates at $z>1$ among the still spectroscopically unidentified population, and control the selection effects (see Sections 7.1.1 and 7.1.2). In total, we ended up with a robust sample of $\sim 150$ objects with photometric redshifts information, and multicolor properties consistent with those of luminous, obscured quasars at $z>1$. This sample should not be considered complete with respect to the X-ray selected, high- $z$ obscured AGN population, but can be considered as representative of the average properties of this class of sources. We compared the average X-ray HR of the high- $z$ obscured AGN candidate sample with the ones derived for the spectroscopically identified samples (Section 7.2). We found that the average $\mathrm{HR}$ for this sample, $\mathrm{HR}=-0.17$, is considerably higher than that derived for spectroscopically classified NL AGNs (HR $=-0.29)$, further confirming that a red observed $R-K$ color $(R-K \gtrsim 5)$ coupled with high-X/O or $v_{24} F_{24} / v_{R} F_{R}$ ratios, is a good indicator for both optical and moderate $\mathrm{X}$-ray obscuration in high-luminosity sources (see also Maiolino et al. 2006).

For the vast majority $(75 \%-80 \%)$ of the high- $z$ obscured AGN candidates, the AGN component is visible in the nearIR (roughly corresponding to the rest-frame $\sim 1-3 \mu \mathrm{m}$ range), where the dust reprocessed emission is expected to dominate the observed SED. These sources would have been selected on the basis of IRAC colors only (see Figures 10 and 11, and Section 7.3). However, at the limiting fluxes of the IRAC COSMOS survey the contamination from non-AGN objects within the selection wedge starts to become important, and the selection criterion would become efficient only when combined with an additional criterion (in this case, the X-ray emission).

We studied the fraction of obscured AGNs in the XMMCOSMOS sample as a function of the $2-10 \mathrm{keV}$ X-ray luminosity, and discussed the differences between optical (spectroscopic and photometric) and X-ray classifications. We confirmed that the fraction decreases with increasing luminosity (see Section 8 and Figure 12), for both the spectroscopically identified and the full sample. At $L_{X} \gtrsim 10^{44} \mathrm{erg} \mathrm{s}^{-1}$, the obscured AGN fraction is of the order of $25 \%-30 \%$ in the $\log L_{X}=44-44.5$ bin and $\sim 15 \%-20 \%$ in the $\log L_{X}=44.5-45$ bin, about a factor of 2-3 lower than the predictions from the Gilli et al. (2007) and Treister \& Urry (2006) models. At lower luminosities, the optical and X-ray classifications largely differ (see green triangles and squares at $\log L_{X}<43 \mathrm{erg} \mathrm{s}^{-1}$ in Figure 12). This may be related, at least in part, to host-galaxy dilution of the intrinsic AGN spectrum, which is expected to be important at low Eddington ratios and at low luminosities (Hopkins et al. 2009).
We studied in detail the SED and the spectral properties of XID 2028 that we consider the prototype of an obscured QSO at high $-z(z=1.592)$ caught in a transition stage from being starburst dominated to AGN dominated, which was possible to isolate only thanks to the combination of X-ray and infrared observations. XID 2028 is one of the brightest XMMCOSMOS sources, with an unabsorbed X-ray luminosity $L_{X} \sim$ $10^{45} \mathrm{erg} \mathrm{s}^{-1}$ and significant $\mathrm{X}$-ray absorption $\left(N_{\mathrm{H}} \sim 10^{22}\right)$, i.e., a Type 2 QSO. The optical spectrum of this source presents strong AGN emission lines ([Ne v] doublet) consistent with our selection method. Moreover, evidence of outflowing material at a velocity of $\sim 300 \mathrm{~km} \mathrm{~s}^{-1}$ (see Figure 13) is also clearly detected from the blueshifted $\mathrm{Mg}$ I and $\mathrm{Mg}$ II complex absorption. The $\mathrm{X}$-ray, optical, and infrared properties and band ratios of XID 2028 are very similar to those predicted by the above mentioned models, and the overall SED is best represented by numerical templates resulting from the models published by Narayanan et al. (2009) to describe $z \sim 2$ DOG (see Figure 14, right panel).

XID 2028 is the brightest object for which such a detailed analysis can be conducted in the XMM-COSMOS catalog. However, as demonstrated in Section 7 , it is reasonable to assume that a large fraction of the sources among the high- $z$ obscured AGN candidates share the same properties, although at lower X-ray $\left(L_{X} \gtrsim 10^{44} \mathrm{erg} \mathrm{s}^{-1}\right)$ and bolometric luminosities. Most of these sources are well within the reach of the Herschel (Pilbratt 2005) instruments between 75 and $500 \mu \mathrm{m}$, which in the GT project in the COSMOS field will reach limiting fluxes of few mJy. Such longer wavelength observations can greatly help in separating nuclear activity and star formation, and assessing the real bolometric output of obscured AGNs. Further investigations, as for example, direct infrared spectroscopy with X-Shooter@VLT (D'Odorico et al. 2004) or LUCIFER@LBT (Mandel et al. 2007) may be crucial in confirming the nature of these candidates.

This work is based on observations obtained with XMMNewton, an ESA Science Mission with instruments and contributions directly funded by ESA Member States and the USA (NASA). In Germany, the XMM-Newton project is supported by the Bundesministerium für Wirtschaft und Technologie/Deutsches Zentrum für Luft- und Raumfahrt (BMWI/DLR, FKZ 50 OX 0001), the Max-Planck Society, and the Heidenhain-Stiftung. Part of this work was supported by the Deutsches Zentrum für Luft-und Raumfahrt, DLR project numbers 50 OR 0207 and 50 OR 0405. In Italy, the XMM-COSMOS project is supported by PRIN/MIUR under grant 2006-02-5203, ASI-INAF grants I/023/05/00, I/088/06 and ASI/COFIS/WP3110,I/026/07/0. This work was supported in part by NASA Chandra grant number GO78136A (F.C., M.E., A.F., H.H.). T.M. acknowledges support from CONACyT 83564 DGAPA/PAPIIT IN10209 to IAUNAM as well as the NASA ADP (NNX07AT02G) grant to UCSD. G.H. and M.S. acknowledge a contribution from the Leibniz Prize of the Deutsche Forschungsgemeinschaft under the grant HA $1850 / 28-1$. N.C. and A.F. were partially supported from a NASA grant NNX07AV03G to UMBC. K.J. is supported by the Emmy Noether-Programme of the German Science Foundation DFG. E.T. is supported by the National Aeronautics and Space Administration through Chandra Postdoctoral Fellowship Award Number PF8-90055. We thank James Aird and Jacobo Ebrero for sending us machine-readable tables of their X-ray luminosity functions, Desika Narayanan for 
help with his model SEDs, and Ryan Hickox for providing unpublished information about the X-Bootes survey. We gratefully thank Nick Wright for a carefully reading of the manuscript. This work is based in part on observations obtained with MegaPrime/ MegaCam, a joint project of Canada-France-Hawaii Telescope (CFHT) and CEA/DAPNIA, at the CFHT, and on data products produced at TERAPIX data center located at the Institut d'Astophysique de Paris. This research has made use of the Keck Observatory Archive (KOA), which is operated by the W. M. Keck Observatory and the NASA Exoplanet Science Institute (NExScI), under contract with the National Aeronautics and Space Administration. We gratefully acknowledge the contribution of the entire COSMOS collaboration; more information on the COSMOS survey is available at http://www.astro.caltech.edu/cosmos. This research has made use of the NASA/IPAC Extragalactic Database (NED) and the SDSS spectral archive. Finally, we thank the anonymous referee for detailed and constructive comments to the first version of this paper.

\section{REFERENCES}

Adelman-McCarthy, J. K., et al. 2006, ApJS, 162, 38

Aird, J., Nandra, K., Georgakakis, A., Laird, E. S., Steidel, C. C., \& Sharon, C. 2008, MNRAS, 387, 883

Aird, J., et al. 2010, MNRAS, 401, 2531

Alexander, D. M., Brandt, W. N., Hornschemeier, A. E., Garmire, G. P., Schneider, D. P., Bauer, F. E., \& Griffiths, R. E. 2001, AJ, 122, 2156

Alexander, D. M., Vignali, C., Bauer, F. E., Brandt, W. N., Hornschemeier, A. E., Garmire, G. P., \& Schneider, D. P. 2002, AJ, 123, 1149

Alexander, D. M., et al. 2005, ApJ, 632, 736

Antonucci, R. R. 1993, ARA\&A, 31, 473

Antonucci, R. R., \& Miller, J. S. 1985, ApJ, 297, 621

Avni, Y., \& Bahcall, J. N. 1980, ApJ, 235, 694

Baldwin, J. A., Philips, M. M., \& Terlevich, R. 1981, PASP, 93, 5

Ballantyne, D. R. 2008, ApJ, 685, 787

Ballantyne, D. R., Everett, J. E., \& Murray, N. 2006, ApJ, 639, 740

Barger, A. J., et al. 2005, AJ, 129, 578

Barkhouse, W. A., \& Hall, P. B. 2001, AJ, 121, 2843

Barmby, P., et al. 2006, ApJ, 642, 126

Bell, E. F., et al. 2004, ApJ, 608, 752

Blanton, M. R., et al. 2003, ApJ, 594, 186

Bondi, M., Ciliegi, P., Schinnerer, E., Smolcic, V., Jahnke, K., Carilli, C., \& Zamorani, G. 2008, ApJ, 681, 1129

Bongiorno, A., et al. 2007, A\&A, 472, 443

Bongiorno, A., et al. 2010, A\&A, 510, 56

Brandt, N. W., \& Alexander, D. M. 2010, Proc. Natl. Acad. Sci., in press (arXiv:1001.5054)

Brandt, N. W., \& Hasinger, G. 2005, ARA\&A, 43, 827

Brandt, N. W., Hornschemeier, A. E., Schneider, D. P., Alexander, D. M., Bauer,

F. E., Garmire, G. P., \& Vignali, C. 2001, ApJ, 558, L5

Brusa, M., et al. 2005, A\&A, 432, 69

Brusa, M., et al. 2007, ApJS, 172, 353 (B07)

Brusa, M., et al. 2009a, ApJ, 693, 8

Brusa, M., et al. 2009b, A\&A, 507, 1277

Caccianiga, A., Severgnini, P., Della Ceca, R., Maccacaro, T., Carrera, F. J., \& Page, M. J. 2007, A\&A, 470, 557

Caccianiga, A., et al. 2008, A\&A, 477, 735

Capak, P., et al. 2007, ApJS, 172, 99

Cappelluti, N., et al. 2007, ApJS, 172, 341

Cappelluti, N., et al. 2009, A\&A, 497, 635 (C09)

Cardamone, C., et al. 2008, ApJ, 680, 130

Ciliegi, P., Zamorani, G., Hasinger, G., Lehmann, I., Szokoly, G., \& Wilson, G. 2003, A\&A, 398, 901

Civano, F., Mignoli, Comastri, A., \& Brusa, M. 2005, MNRAS, 358, 693

Civano, F., et al. 2007, A\&A, 476, 1223

Cocchia, F., et al. 2007, A\&A, 466, 31

Comastri, A., \& Brusa, M. 2008, Astron. Nachr., 329, 122

Comastri, A., Brusa, M., \& Mignoli, M. 2003, Astron. Nachr., 324, 28

Comastri, A., et al. 2002, ApJ, 571, 771

Covey, K. R., et al. 2008, ApJS, 178, 339

Cowie, L. L., Songaila, A., Hu, E. M., \& Cohen, J. G. 1996, AJ, 112, 839
Croton, D. J., et al. 2006, MNRAS, 367, 864

Daddi, E., et al. 2007, ApJ, 670, 156

Della Ceca, R., et al. 2004, A\&A, 428, 383

Della Ceca, R., et al. 2008, A\&A, 487, 119

Dey, A., et al. 2008, ApJ, 677, 943

Di Matteo, T., Springel, V., \& Hernquist, L. 2005, Nature, 433, 604

D'Odorico, S., et al. 2004, Proc. SPIE, 5492, 220

Donley, J. L., Rieke, G. H., Perez-Gonzalez, P. G., \& Barro, G. 2008, ApJ, 687, 111

Ebrero, J., et al. 2009, A\&A, 493, 55

Eckart, M. E., et al. 2006, ApJS, 156, 35

Elvis, M., et al. 1994, ApJS, 95, 1

Elvis, M., et al. 2009, ApJS, 184, 158

Faber, S. M., et al. 2003, Proc. SPIE, 4841, 1657

Faber, S. M., et al. 2007, ApJ, 665, 265

Fabian, A. C., \& Iwasawa, K. 1999, MNRAS, 303, L34

Ferrarese, L., \& Merrit, D. 2000, ApJ, 539, L9

Feruglio, C., et al. 2008, A\&A, 448, 417

Finoguenov, A., et al. 2007, ApJS, 172, 182

Fiore, F., et al. 2003, A\&A, 409, 79 (F03)

Fiore, F., et al. 2008, ApJ, 672, 94

Fiore, F., et al. 2009, ApJ, 693, 447

Franceschini, A., Bassani, L., Cappi, M., Granato, G. L., Malaguti, G., Palazzi, E., \& Persic, M. 2000, A\&A, 353, 910

Frayer, D. T., et al. 2009, AJ, 138, 1261

Gabor, J. M., et al. 2009, ApJ, 691, 705

Gavignaud, I., et al. 2006, A\&A, 457, 79

Gebhardt, K., et al. 2000, ApJ, 543, L5

Gehrels, N. 1986, ApJ, 303, 336

Georgantopoulos, I., Georgakakis, A., \& Akylas, A. 2006, A\&A, 466, 823

Georgantopoulos, I., Georgakakis, A., Rowan-Robinson, M., \& Rovilos, E. 2008, A\&A, 484, 671

Giacconi, R., et al. 2001, ApJ, 551, 624

Gilli, R., Comastri, A., \& Hasinger, G. 2007, A\&A, 463, 79

Gilli, R., et al. 2009, A\&A, 494, 33

Granato, G. L., De Zotti, G., Silva, L., Bressan, A., \& Danese, L. 2004, ApJ, 600,580

Gültekin, K., et al. 2009, ApJ, 698, 198

Hasinger, G. 2008, A\&A, 490, 905

Hasinger, G., Miyaji, T., \& Schmidt, M. 2005, A\&A, 441, 417

Hasinger, G., et al. 2007, ApJS, 172, 29

Hatzminiaoglou, E., et al. 2005, AJ, 129, 1198

Hickox, R. 2009, Chandra Newsletter cover article, Winter 2009, arXiv:0904.3543

Hickox, R., et al. 2009, ApJ, 696, 891

Hopkins, P. F., Hernquist, L., Cox, T. J., Di Matteo, T., Robertson, B., \& Springel, V. 2006, ApJS, 163, 1

Hopkins, P. F., Hernquist, L., Cox, T. J., \& Keres, D. 2008, ApJS, 175, 356

Hopkins, P. F., Hickox, R., Quataert, E., \& Hernquist, L. 2009, MNRAS, 398, 333

Hornschemeier, A. E., et al. 2001, ApJ, 554, 742

Houck, J. R., et al. 2005, ApJ, 622, L105

Ilbert, O., et al. 2009, ApJ, 690, 1236

Ilbert, O., et al. 2010, ApJ, 709, 644

Jahnke, K., et al. 2009, ApJ, 706, L215

Kartaltepe, J., et al. 2010, ApJ, 709, 572

Kauffmann, G., et al. 2003, MNRAS, 346, 1055

Kennicutt, R. C., Jr. 1998, ARA\&A, 36, 189

Kenter, A., et al. 2005, ApJS, 161, 9

Kewley, L. J., Dopita, M. A., Sutherland, R. S., Heisler, C. A., \& Trevena, J. 2001, ApJ, 556, 121

Kim, M., et al. 2007, ApJS, 169, 401

Koekemoer, A. M., et al. 2004, ApJ, 600, L123

Koekemoer, A. M., et al. 2007, ApJS, 172, 196

La Franca, F., \& Cristiani, S. 1997, AJ, 113, 1517

La Franca, F., et al. 2005, ApJ, 635, 864

Lacy, M., et al. 2004, ApJS, 154, 166

Laird, E., Nandra, K., Pope, A., \& Scott, D. 2010, MNRAS, 401, 2763

Lanzuisi, G., Piconcelli, E., Fiore, F., Feruglio, C., Vignali, C., Salvato, M., \& Gruppioni, C. 2009, A\&A, 498, 67

Lawrence, A., \& Elvis, M. 1982, ApJ, 256, 410

Le Floc'h, E., et al. 2007, ApJ, 660, L65

Le Floc'h, E., et al. 2009, ApJ, 703, 222

Lilly, S. J., et al. 2007, ApJS, 172, 70

Lilly, S. J., et al. 2009, ApJS, 184, 218

Loaring, N. S., et al. 2005, MNRAS, 362, 1371

Luo, B., et al. 2010, ApJS, 187, 560 
Luo, B., et al. 2008, ApJS, 179, 19

Lusso, E., et al. 2010, A\&A, 512, 34

Maccaccaro, T., Gioia, I. M., Wolter, A., Zamorani, G., \& Stocke, J. T. 1988, ApJ, 326,680

Mainieri, V., et al. 2005, MNRAS, 356, 1571

Mainieri, V., et al. 2007, ApJS, 172, 368

Maiolino, R., Shemmer, O., Imanishi, M., Netzer, H., Oliva, E., Lutz, D., \& Sturm, E. 2007, A\&A, 468, 979

Maiolino, R., et al. 2006, A\&A, 445, 457

Mandel, H., et al. 2007, Astron. Nachr., 328, 626

Marconi, A., Risaliti, G., Gilli, R., Hunt, L. K., Maiolino, R., \& Salvati, M. 2004, MNRAS, 351, 169

Martinez-Sansigre, A., Rawlings, S., Lacy, M., Fadda, D., Jarvis, M. J., Marleau, F. R., Simpson, C., \& Willott, C. J. 2006, MNRAS, 370, 1479

Martinez-Sansigre, A., Rawlings, S., Lacy, M., Fadda, D., Marleau, F. R., Simpson, C., Willott, C. J., \& Jarvis, M. J. 2005, Nature, 436, 666

McCracken, H., et al. 2010, ApJ, 708, 202

Menci, N., Fiore, F., Puccetti, S., \& Cavaliere, A. 2008, ApJ, 686, 219

Merloni, A. 2004, MNRAS, 353, 1035

Merloni, A., et al. 2010, ApJ, 708, 137

Mignoli, M., et al. 2004, A\&A, 418, 827

Moran, E. C., Filippenko, A. V., \& Chornock, R. 2002, ApJ, 579, L71

Murray, S. S., et al. 2005, ApJS, 161, 1

Nandra, K., et al. 2002, ApJ, 576, 625

Narayanan, D., et al. 2009, MNRAS, 400, 1919

Norman, C., et al. 2002, ApJ, 571, 218

Perola, G. C., et al. 2004, A\&A, 421, 491

Pilbratt, G. 2005, Proc. SPIE, 4850, 586

Polletta, M., et al. 2007, ApJ, 663, 81

Pozzi, F., et al. 2007, A\&A, 468, 603

Prescott, M. K. M., Impey, C. M., Cool, R. J., \& Scoville, N. Z. 2006, ApJ, 644, 100

Puccetti, S., et al. 2009, ApJS, 185, 586

Ranalli, P., Comastri, A., \& Setti, G. 2003, A\&A, 399, 39

Richards, G. T., et al. 2002, AJ, 123, 2945

Sacchi, N., et al. 2009, ApJ, 703, 1778

Sajina, A., Lacy, M., \& Scott, D. 2005, ApJ, 621, 256

Salvato, M., et al. 2009, ApJ, 690, 1250

Sanders, D. B., Soifer, B. T., Elias, J. H., Neugebauer, G., \& Matthews, K. 1988, ApJ, 328, 35

Sanders, D., et al. 2007, ApJS, 172, 86
Schinnerer, E., et al. 2007, ApJS, 172, 46

Scoville, N., et al. 2007a, ApJS, 172, 1S

Scoville, N. Z., et al. 2007b, ApJS, 172, 38

Severgnini, P., et al. 2003, A\&A, 406, 483

Severgnini, P., et al. 2005, A\&A, 431, 87

Severgnini, P., et al. 2006, A\&A, 451, 859

Shapley, A. E., Steidel, C. C., Pettini, M., \& Adelberger, K. L. 2003, ApJ, 588, 65

Sijacki, D., Springel, V., Di Matteo, T., \& Hernquist, L. 2007, MNRAS, 380, 877

Silk, J., \& Rees, M. 1998, A\&A, 331, L1

Silverman, J. D., et al. 2008, ApJ, 679, 118

Silverman, J. D., et al. 2009a, ApJ, 696, 396

Silverman, J. D., et al. 2009b, ApJ, 695, 171

Spergel, D. N., et al. 2003, ApJS, 148, 175

Stalin, C. S., Petitjean, P., Srianand, R., Fox, A. J., Coppolani, F., \& Schwope, A. 2010, MNRAS, 401, 294

Stern, D., et al. 2005, ApJ, 631, 163

Sutherland, W., \& Saunders, W. 1992, MNRAS, 259, 413

Szokoly, G. P., et al. 2004, ApJS, 155, 271

Taniguchi, Y., et al. 2007, ApJS, 172, 9

Tozzi, P., et al. 2006, A\&A, 451, 457

Treister, E., \& Urry, C. M. 2006, ApJ, 652, L79

Treister, E., et al. 2005, ApJ, 621, 104

Treister, E., et al. 2009a, ApJ, 693, 1713

Treister, E., et al. 2009b, ApJ, 706, 535

Trump, J. R., et al. 2007, ApJS, 172, 383

Trump, J. R., et al. 2009, ApJ, 696, 1195

Ueda, Y., Akiyama, M., Ohta, K., \& Miyaji, T. 2003, ApJ, 598, 886

Urry, C. M., \& Padovani, P. 1995, PASP, 107, 803

Veilleux, S., et al. 2009, ApJS, 182, 628

Vignali, C. 2001, PhD thesis, Bologna Univ.

Vignali, C., Comastri, A., Fiore, F., \& La Franca, F. 2001, A\&A, 370, 900

Vignali, C., et al. 2009, MNRAS, 395, 2189

Weiner, B. J., et al. 2009, ApJ, 692, 187

Wolf, C., et al. 2004, A\&A, 421, 913

Yan, L., et al. 2005, ApJ, 628, 604

Yan, L., et al. 2007, ApJ, 658, 778

Yencho, B., Barger, A. J., Trouille, L., \& Winter, L. M. 2009, ApJ, 698, 380

Zamojski, M. A., et al. 2007, ApJS, 172, 468

Zombeck, M. V. 1990, Handbook of Space Astronomy and Astrophysics (Cambridge: Cambridge Univ. Press) 